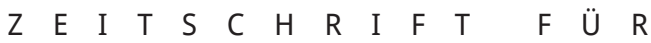

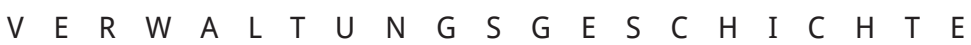

B A N D 2, 200717

S E I T E $43-67$

D O I : $10.2478 / A D H I-2018-0015$

\title{
(Um)Ordnen des Grenzraums zwischen dem Salzburger und Tiroler Herrschaftsbereich im Zillertal im 18. und 19. Jahrhundert
}

\author{
ELLINOR FORSTER
}

Zwischen dem Erzstift Salzburg ${ }^{1}$ und der Grafschaft Tirol ${ }^{2}$ stießen bis 1816 Pfleg- und Landgerichte aneinander, die mehr oder weniger deutlich voneinander abgegrenzt waren. Die Charakteristik, die Vilém Flusser jeder Grenze attestiert, keine klare Linie, sondern eine "verworrene Zone « zu sein, in der sich Phänomene kreuzen und vermischen, ${ }^{3}$ liegt bei territorialen Grenzen im Mittelalter und in der Frühen Neuzeit noch sehr deutlich auf der Hand. Die Herrschaftsgewalt konzentrierte sich in erster Linie an den Höfen, die sich im Inneren der Territorien befanden, und definierte sich nicht über ein exakt umrissenes Land, sondern durch erworbene Rechte - über Untertanen und auf Grundbesitz mit den jeweils damit verbundenen Abgaben. Grundbesitz wurde strategisch oftmals an verschiedenen Orten angestrebt, um Zugang zu möglichst vielfältigen Ressourcen zu erhalten. Daraus resultierten eine starke Streuung und ein vielfaches Ineinandergreifen der Territorien. Grenzräume stellten sich auf diese Weise sehr offenkundig als "graue Zonen« dar, die eng miteinander verflochten waren. ${ }^{4}$

Das lässt sich sehr gut am Beispiel des Zillertals nachzeichnen, das in der Frühen Neuzeit zwischen Tirol und Salzburg herrschaftlich geteilt war. Im größeren
Herrschaftsverband des Herzogtums Bayern hatten die Erzbischöfe von Salzburg seit dem Frühmittelalter große Komplexe an Grundbesitz im hinteren und mittleren Talbereich erworben, die jedoch nicht direkt aneinander grenzten. Verwaltet wurde dieses salzburgische Zillertal zunächst von einem Pfleger, der auf der Burg Kropfsberg am Taleingang saß, deren Burgfrieden wiederum territorial nicht an das übrige Gebiet anschloss. Im 16. Jahrhundert kam es zur Verlegung des Gerichtssitzes in den Ort Zell, der im Talinneren lag, und in der zweiten Hälfte des 17. Jahrhunderts zur zusätzlichen Erhebung des vorderen Teils zu einem eigenen Pfleggericht mit dem Gerichtssitz Fügen. Zwischen den beiden Salzburger Gerichten, links des namensgebenden Flusses Ziller, gehörten drei Dörfer zum Tiroler Gericht Rottenburg. Dessen Verwaltungssitz befand sich während des gesamten Untersuchungszeitraums am Taleingang. Als sich Tirol im 13. und Salzburg im 14. Jahrhundert aus dem Herzogtum Bayern herausgelöst und eine eigene Herrschaftsgewalt aufgebaut hatten, grenzten auf dem Boden des Zillertals die drei Territorien Salzburg, Tirol und Bayern aneinander, denn eine Hofmark und einzelne Dörfer auf der rechten Seite des Zillers gehörten zum bayerischen 
Gericht Rattenberg. Mit der Einnahme dieses Gerichts durch die Habsburger zu Beginn des 16. Jahrhunderts gelangte auch der ehemals bayerische Anteil im Zillertal an Tirol, sodass sich nunmehr auch auf der rechten Talseite - wiederum zwischen den Salzburger Gerichten gelegen - Tiroler Territorium befand. ${ }^{5}$

Mit diesem räumlichen Ineinandergreifen des Territoriums sind die "struppigen Mengen ${ }^{6}$ des Grenzbereichs, also die grenzübergreifenden, sich einer eindeutigen Zuordnung entziehenden Phänomene, jedoch noch nicht ausreichend beschrieben. Flusser macht bei Grenzen Elemente aus, die sowohl zum einen wie zum anderen Feld gehören. Bei `modernen`Grenzen denkt er dabei an »weiße Stellen «, an ein »no-man’s-land « zwischen den Grenzlinien. ${ }^{7}$ In historischen Grenzräumen konnten sich auch die rechtlichen Zuständigkeiten der beiden Herrschaftsgewalten vermischt entwickeln. So hatten beispielsweise im Zillertal mit der Entdeckung und dem Abbau von Metallvorkommen die beiden Landesfürsten im 16. und 17. Jahrhundert Verträge geschlossen, in denen sie sich auf eine gemeinsame Verwaltung und Nutzung dieser Erzvorkommen einigten. Das dafür notwendige Holz befand sich vor allem im Salzburger Teil, das somit auch von Tirolern geschlagen werden konnte. Über weitere Verträge, die über die Belange des Zillertals hinausgingen, sicherten sich die Erzbischöfe von Salzburg zudem die Durchfuhr von Salz durch habsburgisches Territorium gegen die Nutzung der Zillertaler Wälder durch Tirol auch für dessen eigene Bergwerke. ${ }^{8}$ Dazu kam eine Zusammenarbeit im rechtlichen Bereich. Obwohl die Erzbischöfe von Salzburg in ihrem Gebiet die hohe Gerichtsbarkeit ausüben konnten, wurden die im Zillertal zum Tod verurteilten Personen zur Hinrichtung an die angrenzenden Tiroler Gerichte übergeben. ${ }^{9}$ Da alle Bewohner und Bewohnerinnen die einzige fahrbare Straße nutzten, welche über die unterschiedlichen Herrschaftsgebiete hinweg durch das Tal führte, war auch die Zuständigkeit für die Erhaltung dieser Straße eine geteilte. ${ }^{10}$ Unabhängig von den Herrschaftsgrenzen, die quer über den Fluss verliefen, bildete schließlich der Ziller selbst eine weitere Grenze, nämlich jene zwischen den Diözesen Salzburg und Brixen.

Die Überlegungen von Flusser sind gerade darum spannend, weil sie diese Eigenschaften nicht den offenkundig vage definierten historischen Grenzräumen, eine klare Trennung von Gebieten zu vollziehen, zusprechen. Diese würden neben der Trennung immer zugleich auch eine Verbindung herstellen, indem die zuständigen Personen beispielsweise über ihre Wahrung miteinander in Kontakt treten müssen. ${ }^{11}$ Dasselbe Phänomen lässt sich am Beispiel der Herrschaftsgrenzen im Zillertal in der zweiten Hälfte des 18. Jahrhunderts beobachten. Ab den 1760er Jahren setzte eine intensive Phase der Kommunikation zwischen den Vertretern beider Territorien ein, da man die herrschaftliche Vermischung des Grenzraumes entflechten wollte. Für die bisher so unklare Abgrenzung brachte beispielsweise der Salzburger Pfleger Gottfried von Moll 1780 nur noch Unverständnis auf. Mit Bezug auf den letzten Vertrag zwischen Salzburg und Tirol von 1690 schrieb er, dass man glauben könne,

es wäre allen Gränz-Differenzen auf das Beste vorgebeugt, die Jurisdiction ausser aller Zweydeitigkeit gesetzt, und weiter nichts vonnöthen, liest man aber in diesem Vertrag mit Bedacht ein wenig weiter fort, so findet man gleich, wie der Vertrag selbst jene Gränzen, die er anfangs mit einer richtigen Linie deutlich unterscheidet, mit nachgefolgten Klausuln wiederum in die größte Verwirrung bringt, dort ein Gut, da mehrere Gütter, dort wieder eine Alpe von der Jurisdiction, die die Gränzen Linie giebt, eximirt. [...] Nimmermehr hätte man wohl die zweyerlei Jurisdictionen mehr durcheinander weben können, ausser man hätte auch in den Häußern, Stuben, Kämern, Küche, u. Stallung, oder auf den Feldern die Zuwäge, u. Raine von den Äckern eben also abgetheilt. ${ }^{12}$

Hinter den Verhandlungen über die Bereinigung von Rechten, die Verlegung des Grenzverlaufs, um die territorialen Einschlüsse im jeweils anderen Gebiet zu beseitigen, über den Austausch ganzer Gerichte stand die längerfristige Entwicklung eines neuen Verständnisses von Herrschaftsraum als geografisch geschlossenes politisches Territorium, das sich vermessen und darstellen sowie bis an die Grenzen gestalten und somit regieren ließ. ${ }^{13}$ So begannen etwa auch in den 1750 er Jahren in den österreichischen Ländern die Erhebungen zu den Seelenkonskriptionen, die die Schwierigkeit, dass Grund und Untertanen nicht immer eindeutig zuzuordnen waren, augenscheinlich machten. ${ }^{14}$ Das zeigte sich etwa 
auch im Zillertal, als in den beiden Dörfern Gagering und Unterahrnbach, in denen sich die Zugehörigkeit jeweils nach Haus und Grund unterschied, die Häuser in die österreichische Zählung einbezogen wurden und dies entsprechenden Protest hervorrief - mit dem Wissen, dass diese sichtbare Zugehörigkeitsmarkierung der aufgemalten Hausnummern Folgen in Hinsicht einer Konskription oder Steuerpflicht nach sich ziehen konnte. ${ }^{15}$ Auch dass diese Prozesse nicht linear und einseitig von den Herrschaftsträgern bestimmt verliefen, ${ }^{16}$ belegen die Entwicklungen im Zillertal. Die konkreten Tausch- und Ausgleichspläne, welche auch die anderen Grenzgerichte zwischen Tirol und Salzburg miteinbezogen, wurden nie völlig in die Tat umgesetzt, sondern stagnierten zwischendurch und kamen nicht zuletzt durch die Kriege ab den 1790er Jahren und die sich verändernden Herrschaftsverhältnisse nach 1800 immer wieder zum Erliegen. Schließlich erübrigten sie sich 1816, als Tirol und Salzburg gemeinsam zu Österreich gehörten und das gesamte Zillertal Tirol zugesprochen wurde.

Im Kontext dieser Verhandlungen waren für die Herrschaftsvertreter der genaue Verlauf der Grenze und mögliche unrechtmäßige Übergriffe immer bedeutender geworden. Je konkreter man allerdings die Grenze festlegen oder ihrem Verlauf nachspüren wollte, desto vager und schwerer greifbar schien sie trotz einer vermeintlich klar definierten Linie zu werden. In diesem Ringen um die Übereinstimmung von Idealvorstellung und an Ort und Stelle wahrgenommenem Raum wurde auch auf die Raumbilder und das Raumwissen der Talbewohner zurückgegriffen. Diese sollten Auskunft über Grenzverläufe und Praktiken geben. Damit wird offensichtlich, welch zentrale Rolle die Kommunikation bei der Raumkonstruktion spielt. Folgt man dem Ansatz von Martina Löw, lässt sich die Entstehung des Raums durch die »(An)Ordnung" von (sozialen) Objekten im Raum und deren Verknüpfung beschreiben. Dadurch werde eine relationale und somit stets veränderbare Raumordnung strukturiert, wenn diese auch durch die Etablierung von Institutionen zur Verstetigung neigt. ${ }^{17}$ Dem fügt Gabriela B. Christmann konstruktivistische Kommunikationsansätze hinzu, um den Vorgang dieses Anordnens und Verknüpfens genauer fassen zu können. Personen handeln demnach in einem räumlichen Umfeld, das sie sich auf der Grundlage ihrer Körperlichkeit sowie Sinnesausstattung erschließen und es als einen sie umgebenden Raum auffassen. Die wahrgenommenen Gegebenheiten, Objekte und Subjekte, deren Handlungsweisen sowie soziale Ordnungen werden daraufhin diesem Raum zugeordnet, ihm zugleich bestimmte Bedeutungen zugeschrieben und auf dieser Grundlage raumbezogene Wirklichkeitsdeutungen und Handlungsweisen entwickelt. Dabei steht die subjektive Raumwahrnehmung in ständigem kommunikativem Austausch mit jener anderer Personen, indem im Raum agiert und/oder über ihn gesprochen wird. Durch das Handeln kommt es zur (Re)Produktion von Strukturen sowie in Form regelmäßig ablaufender Handlungsvollzüge zu einer Verstetigung und zur Herstellung eines Orientierungsrahmens für das weitere Handeln. ${ }^{18}$

Damit liegt der Blick nicht nur auf dem Sprechen über den Raum, sondern auch auf dem kommunikativen Handeln im Sinn der Raumnutzung, um Aufschlüsse über Raumkonstruktionen zu erhalten. ${ }^{19}$ Das Handeln der vor allem agrarisch geprägten wie auch vom Bergbau lebenden Talbevölkerung konstruierte und verfestigte die sozialen, rechtlichen und wirtschaftlichen Strukturen mit all ihren Hierarchien aus ihrer Perspektive. Die Pfarrgemeinde ging in die Kirche, nahm teil an Prozessionen und Wallfahrten, wo sie auf die kirchlichen Amtsvertreter traf, denen die Wissensmacht und damit Weisungsmacht in religiösen Praktiken für ein sgottgefälliges Leben zugeschrieben wurde. Ihre Steuern sowie grundherrschaftlichen und kirchlichen Abgaben lieferten die Menschen an bestimmten Orten ab. Dabei traten sie persönlich oder über die sogenannten Ausschüsse - gewählte Männer, die das jeweilige Dorf vertraten - mit den Herrschaftsvertretern in Kontakt. Diese Ausschüsse bewegten sich auf andere Weise im Raum, sie waren mit einer gewissen politischen Handlungsmacht ausgestattet und hatten direkteren Zugang zu den Herrschaftsträgern. Davon abgesehen stand die Bevölkerung auch untereinander in einem Geflecht von sozialen Beziehungen, die sich im Raum anordneten, wie etwa Wirtshausbesuche, Kirchtagsfeiern, Hochzeiten, Taufen und Begräbnisse oder das Schließen und Lösen von Arbeitsverhältnissen. Dabei sind Raumwissen und Raumhandeln kaum voneinander zu trennen.

Für fast jegliches Tun außerhalb des eigenen Dorfes wurden Herrschafts- und Diözesangrenzen ebenso mühelos wie die Grenzen des eigenen Grundes überschritten. Da dieses Überqueren der Herrschaftsgrenzen für die Erledigung der Agenden kaum Probleme bereitete, war es 
auch nicht notwendig, alternative Wege zur Hauptstraße durch das Tal, etwa aus Salzburger Perspektive über die Pässe zum direkt angrenzenden Hauptland, auszubauen. Nur in Krisensituationen, wie etwa bei längerfristigen Sperren von Lebensmitteln, wurde dies angedacht. Die sich überlappenden Herrschafts-, Diözesan- und Wirtschaftsräume standen für die Bevölkerung offensichtlich nicht miteinander in Konflikt, wurden wahrscheinlich nicht einmal als unterschiedlich, sondern vielmehr durch das Bündel des alltäglichen Raumhandelns entlang der Erledigung ihrer Vorhaben als ein einziger mehrfach strukturierter Raum wahrgenommen. Die Menschen differenzierten zwar zwischen >Tirolern` und 'Salzburgern`, aber vielleicht war dies so zu verstehen, wie sich mit jeglicher Unterscheidung, auch sozialer Gruppen, verschiedene Handlungsoptionen und Zuschreibungen verbanden.

Die beschrittenen Wege und damit Raumnutzungen der Talbevölkerung waren eng mit dem Verwaltungshandeln der Herrschaftsvertreter verwoben, die sich als Hüter und zugleich Schöpfer der Ordnung verstanden. Ihre Raumpraktiken in diesen Grenzgerichten orientierten sich über die üblichen Agenden hinaus auch an ihrer Aufgabe, den Hoheitsbereich zu wahren und keine Eingriffe zu dulden. Neben dem Besuch von Kirche und Wirtshaus durchmaßen sie den Raum in erster Linie durch Kommunizieren mittels Briefen, etwa mit dem Herrschaftsvertreter des Nachbargerichts und vor allem mit den übergeordneten Institutionen - der Repräsentation und Kammer beziehungsweise ab 1763 dem Gubernium $^{20}$ auf Tiroler sowie dem Hofrat und dem Erzbischof auf Salzburger Seite. ${ }^{21}$ Sie ließen über Gerichtsboten oder Schreiber Untertanen zum Verhör in das Gerichtsgebäude bestellen oder nahmen deren Anliegen und Berichte auf, wenn diese aus eigener Initiative dort erschienen, begaben sich manchmal aufgrund verschiedener Vorkommnisse selbst sauf Augenschein ‘ oder trafen sich - in wenigen Fällen - persönlich mit dem Herrschaftsvertreter des anderen Gerichts zur Aushandlung eines Konflikts. Dabei agierten sie im Namen ihrer Herrschaft, können jedoch keineswegs als `der Staat ‘ begriffen werden, wie sich diese Gleichsetzung in der älteren Forschung oftmals findet. ${ }^{22}$ Schaut man genau auf ihr Vorgehen, treten deutlich ihre eigenen Ziele und Strategien zutage. Die beiden eng verflochtenen Territorien abhängig von den jeweils konkret handelnden Personen eine Dynamik entstehen lassen, die durch Misstrauen und Konkurrenz geprägt war. Kritisch beäugten sie das gegenseitige Tun, hinterfragten dessen Legitimität und richteten ihr Handeln darauf aus. Dabei war der Blick stets auf den Raum gerichtet.

Wenn innerhalb des, wie oben beschrieben, als relational aufeinander bezogen verstandenen Raumgefüges plötzlich Änderungen vorgenommen wurden, gerieten die Raumvorstellungen aller Beteiligten in Konflikt. Dies führte in der Folge in verdichteter Kommunikation zu Reaktionen und Aushandlungen und zum Versuch, das eigene Idealbild des Raumes wieder herzustellen oder es neu anzupassen. Die Handlungsmacht, in diesen Raum(an)ordnungen Veränderungen vorzunehmen, verteilte sich entlang der Hierarchien in ungleicher Weise. Die Herrschaftsvertreter konnten über Befehle stärker in das Raumgefüge eingreifen und dieses umordnen - dies jedoch keineswegs willkürlich. Sie mussten ihr Vorgehen von höherer Stelle absegnen lassen. Die Notwendigkeit der Legitimierung tritt besonders deutlich zutage, wenn ihnen etwa vom Herrschaftsvertreter des Nachbarterritoriums das Recht zu den jeweiligen Anordnungen abgesprochen wurde. Auch den Untertanen standen durch das Einreichen von Gravamina bei den zentralen Behörden Handlungsoptionen offen, wenn sie das Verhalten der Herrschaftsvertreter als unangemessen befanden. Durch das Ausführen oder bei geringerem Druck auch das Umgehen oder Anpassen der Befehle veränderten sie ihre Raumkonstruktion.

Da sich Raum immer auch über Grenzen, die konkreter oder vager sein konnten, definiert, wird im Folgenden von den Herrschaftsgrenzen ausgegangen und in drei Annäherungen untersucht, welche Bedeutung diese für die Raumkonstruktion der Akteure und Akteurinnen hatten. Damit liegt der Hauptfokus auf dem ersten der drei in diesem Band fokussierten Aspekte, der 'Raumbildung durch Grenzen`. Zunächst geht es um die Frage, welche Relevanz der Sichtbarkeit der Grenzen zukam. Seit dem beginnenden 17. Jahrhundert befanden sich durchgehende Grenzmarkierungen im Zillertal. Die ständige Überschreitung der Grenzen durch die Talbevölkerung könnte den Schluss nahelegen, dass diese in erster Linie nur für die Herrschaftsvertreter aufgrund ihrer Aufgabe, über sie zu wachen, wichtig gewesen seien. Tatsächlich begannen diese Grenzmarkierungen im 
Kontext der Ausgleichsverhandlungen, wie erwähnt, auch eine immer bedeutendere Rolle zu spielen. Blickt man jedoch - im zweiten und dritten Schritt - anhand von Beispielen aus der Mitte des 18. und aus dem beginnenden 19. Jahrhundert konkreter auf einzelne Grenzabschnitte und verknüpft diese mit der >Raumbildung durch Nutzung`, also den damit einhergehenden Handlungsmöglichkeiten und Handlungsvorschriften, dann zeigt sich, dass die Grenzen für die Raumkonstruktion verschiedener Akteursgruppen konstitutiv waren und je nach Eingriff und Umordnung die Raumvorstellungen verändern konnten.

Die deutschsprachige Geschichtswissenschaft in Österreich und Deutschland beschäftigt sich seit etwa 20 Jahren intensiv mit dem Phänomen Grenze. Am Beginn standen Studien, die vom regierenden Zentrum in Richtung Grenze blickten, wie etwa die umfassenden Untersuchungen in dem Sammelband "Grenze und Staat «, den Waltraud Heindl und Edith Saurer 2000 herausgaben. ${ }^{23}$ Im Kontext der Grenzöffnungen in Ostmitteleuropa nach 1989 rückte auch zugleich das Interesse an den Grenzräumen selbst mit der Frage nach Identitäten und Zugehörigkeiten in den Mittelpunkt und regte eine Reihe von Untersuchungen zu den Grenzen der ehemaligen Habsburgermonarchie an. ${ }^{24}$ Aus der Perspektive der Bundesrepublik Deutschland standen vor allem deren Grenzen zu Frankreich und zu Polen im Zentrum der Forschung. ${ }^{25}$ Über den Zusammenhang der Teilungen und der Geschichte Polens seit dem 18. Jahrhundert trafen sich die Studien wiederum mit der österreichischen Forschungsperspektive und wurden zugleich auf den Ostseeraum ausgeweitet. ${ }^{26}$

Das anhaltende Interesse am Thema `Grenze` führte in der Geschichtswissenschaft in den letzten Jahren dazu, dass der Gegenstand auf Untersuchungen aller Arten von Grenzen ausgedehnt wurde - gefragt wird inzwischen nach Grenzen zwischen gesellschaftlichen Gruppen, zwischen den Geschlechtern, zwischen Auffassungen oder Sprachen. ${ }^{27}$ Der Fokus lag dabei insbesondere auf der Frage nach Verflechtungen im Grenzraum beziehungsweise an den Übergängen. ${ }^{28}$ Überlegungen zur Konstruktion von Raum wurden bisher vor allem im Kontext von >mental maps gestellt,, ${ }^{29}$ wohingegen sich die Anwendung raumsoziologischer Modelle auf die Erforschung von historischen Grenzräumen noch weniger konsequent und umfassend durchgesetzt hat. ${ }^{30}$

\section{Sichtbarkeit der Grenzen als Gradmesser für die Raumkonstruktion?}

Als ab den 1760er Jahren die Zeichen auf Entflechtung des Raums und deutlichere Abtrennung der aneinander grenzenden herrschaftlichen Gerichte standen, war es keineswegs so, dass der Verlauf der Grenzen noch nicht klar bestimmt oder nicht deutlich sichtbar gewesen wäre. Einzelne Markierungen im Raum gab es schon seit dem Spätmittelalter. Ein erster Grenzstein findet sich etwa im Zillertaler Landrecht ${ }^{31}$ aus dem 15. Jahrhundert bei der Nennung des Grenzverlaufs erwähnt, der sonst an markanten Felsen, Hügeln und Bächen entlang beschrieben wurde. ${ }^{32}$ Ein Vertrag von 1533 hatte die Markierung der Jagdgebiete des Erzbischofs von Salzburg und des Landesfürsten von Tirol im Zillertal festgelegt, die mit den jeweiligen Wappen gekennzeichnet werden sollten. Diese Abgrenzung hatte nur für die Fürsten beziehungsweise deren Herrschaftsvertreter, die im Fall von Salzburg ebenfalls dort jagen durften, eine Bedeutung. Im Kontext neuerlicher Vertragsaushandlungen verständigte man sich 1690 auf eine deutlicher sichtbare Markierung der Herrschaftsgrenzen, die schließlich 1711 umgesetzt wurde. ${ }^{33}$ Schon zuvor hatten Karten des 17. Jahrhunderts auf dem Papier die Grenzen mit einer Linie gezogen, nun sollte das auch im Raum nachvollziehbar sein. Mit dem Materialisieren einer Grenze ging das Bestreben nach Vergewisserung und Verbindlichkeit einher, die zwischen den Herrschaftsräumen stets wieder neu herzustellen waren. Daher war das Setzen und Kontrollieren von Markierungen immer als ein Unternehmen gedacht, das von den Bevollmächtigten beider Herrschaftsräume begleitet und genau überwacht werden sollte.

Allerdings scheint sich das Bedürfnis nach einer deutlichen Sichtbarkeit der Grenze nur auf den Talbereich bezogen zu haben. Hier wurden extra dafür angefertigte Markierungssteine aufgestellt, die mit den Buchstaben >T` und 'S s für Tirol und Salzburg sowie der Jahreszahl 1690 auf den verbindlichen Vertrag verwiesen. Je weiter oben am Berg die Grenze verlief, desto mehr genügten die schon in den alten Quellen genannten Berggipfel und Berggrate sowie Bachverläufe als Markierungspunkte. Alle diese Punkte wurden durch Nummerierung systematisch geordnet, die zwar nicht an den Steinen und den anderen Markierungsorten selbst 
ersichtlich, aber in begleitenden Dokumenten vermerkt war. Damit ergab sich zugleich die Leserichtung der Grenze und des Raums für diejenigen, die Einblick in diese Dokumente hatten.

Mit den Verhandlungen über die Angleichung der Grenzen oder den Austausch von Gebieten intensivierte sich zunächst die Kartenproduktion. Die alten Darstellungen aus dem 17. Jahrhundert, mit denen die Herrschaftsvertreter bisher in Konfliktfällen argumentiert hatten, scheinen nicht mehr ausgereicht $\mathrm{zu}$ haben. Beide Landesherrschaften ließen zeitgleich genauere Karten erstellen, die zudem nicht nur die Landesgrenzen darstellten, sondern im Versuch, die gegebenen Hoheits- und Rechtsverhältnisse in ihrer Gesamtheit abzubilden, auch die Waldnutzungsgrenzen und die alten Jagdgrenzen miteinbegriffen (Abb. 1). ${ }^{34}$ Damit hatten sich auf der Karte unter dem genauen Blick die Grenzlinien verdreifacht und waren diffuser geworden, statt eindeutig zu bleiben wie auf den alten Karten. Auch diese beiden 1766 fertig gestellten Karten wurden innerhalb weniger Jahre als nicht mehr brauchbar eingestuft. Der Versuch, sich daraufhin auf eine gemeinsame Kartenerstellung $\mathrm{zu}$ einigen, gestaltete sich $\mathrm{zu}$ einem sehr von gegenseitigem Misstrauen begleiteten Prozess. Zudem fiel das Tiroler Kartierungsunternehmen von Peter Anich und Blasius Hueber, die mit der Triangulierung eine neue Methode anwandten, genau in diese Zeit. Die Salzburger Proteste nach der Veröffentlichung dieses "Tyrol-Atlas « zeigen deutlich, welche Bedeutung Landesfürsten der Darstellung von Grenzverläufen und damit der Zeichnung des Raumes - im Sinne einer Wiedergabe von >Realität ‘ - zumaßen. ${ }^{35}$

Im erwähnten Vertrag von 1690, der die Sichtbarmachung der Grenze durch Markierungssteine festgelegt hatte, war auch vereinbart worden, dass der Grenzverlauf nach dem Einsetzen der Steine alle acht Jahre von Vertretern beider Herrschaften gemeinsam abgegangen und kontrolliert werden solle. ${ }^{36}$ Das hätte eine kontinuierliche Kommunikation der Beamten über die Grenzund damit Raumbeschaffenheit mit sich gebracht. Doch offensichtlich fanden diese gemeinsamen Begehungen nicht statt. Zumindest existieren keine Protokolle oder Berichte darüber, und in den 1790er Jahren wurde rückblickend hinsichtlich aller Grenzverläufe zwischen Salzburg und Tirol beklagt, dass man diese Pflicht nicht wahrgenommen und vernachlässigt habe. ${ }^{37}$ Über die
Gründe lassen sich nur Vermutungen anstellen. Vielleicht war der genaue Verlauf der Grenzen auch den Herrschaftsvertretern in den ersten Jahrzehnten des 18. Jahrhunderts nicht so wichtig, oder sie betrachteten die Abgrenzung mit dem Einsetzen der Steine als endgültig geregelt. Eine Rolle spielte im Zillertal ab den 1750er Jahren wahrscheinlich auch der Umstand, dass das Verhältnis zwischen den Pflegern von Fügen und Rottenburg, also zwischen Salzburg und Tirol, sehr gespannt war und man sich daher vielleicht nicht auf eine solche gemeinsame Unternehmung verständigen konnte oder wollte.

Ein erstes Grenzbegehungsprotokoll datiert aus dem Jahr 1793. Dieser Zeitpunkt steht mit den Ausgleichsverhandlungen in engem Zusammenhang. Vier Jahre zuvor war in Innsbruck der Registraturdirektor Gassler damit beauftragt worden, alle Verträge und Karten zu den `Grenzirrungen` zwischen Tirol und Salzburg durchzusehen und auszuwerten. Aufgrund der spezifischen Verwaltungsstruktur gestaltete sich dieses Unternehmen sehr schwierig. Während in Salzburg die Kommunikation zwischen den Pfleggerichten und dem Hofrat beziehungsweise Erzbischof immer sehr rasch erfolgen konnte und daher die Materialien auch nicht an so vielen verschiedenen Orten zu liegen kamen, war der österreichische bürokratische Weg ein differenzierterer. Die Informationen und Unterlagen gingen von den Gerichten nach Innsbruck beziehungsweise nach der Etablierung der Kreisämter 1754 nach und nach auch zuerst über diese Instanz zur zentralen Behörde in Innsbruck, und von dort an die Hofstellen in Wien. Daher hatten die Tiroler Verhandelnden stets Schwierigkeiten, die Gesamtheit der Verträge und existierenden Karten zu überblicken. Für seine Arbeit wurden Gassler 23 Kisten aus Wien geschickt, ${ }^{38}$ nach mehrmaligen Verlängerungsansuchen legte er 1793 schließlich neun Bände mit genauen Beschreibungen aller Grenzgerichte zwischen Tirol und Salzburg vor, die Gegenstand der Verhandlungen waren. Um vonseiten Salzburgs darauf reagieren zu können, wurde im Herbst 1793 die "Gränz Beschau dermalen privative«, also ohne Hinzuziehung von Tiroler Herrschaftsvertretern, vorgenommen. ${ }^{39}$

Diese Grenzabschreitung, die 1798 nochmals wiederholt wurde, ${ }^{40}$ brachte eine Reihe von Ungereimtheiten zutage, die mehr verwirrten als Eindeutigkeit beförderten und auf verschiedene Raumkonzeptionen verwiesen. 


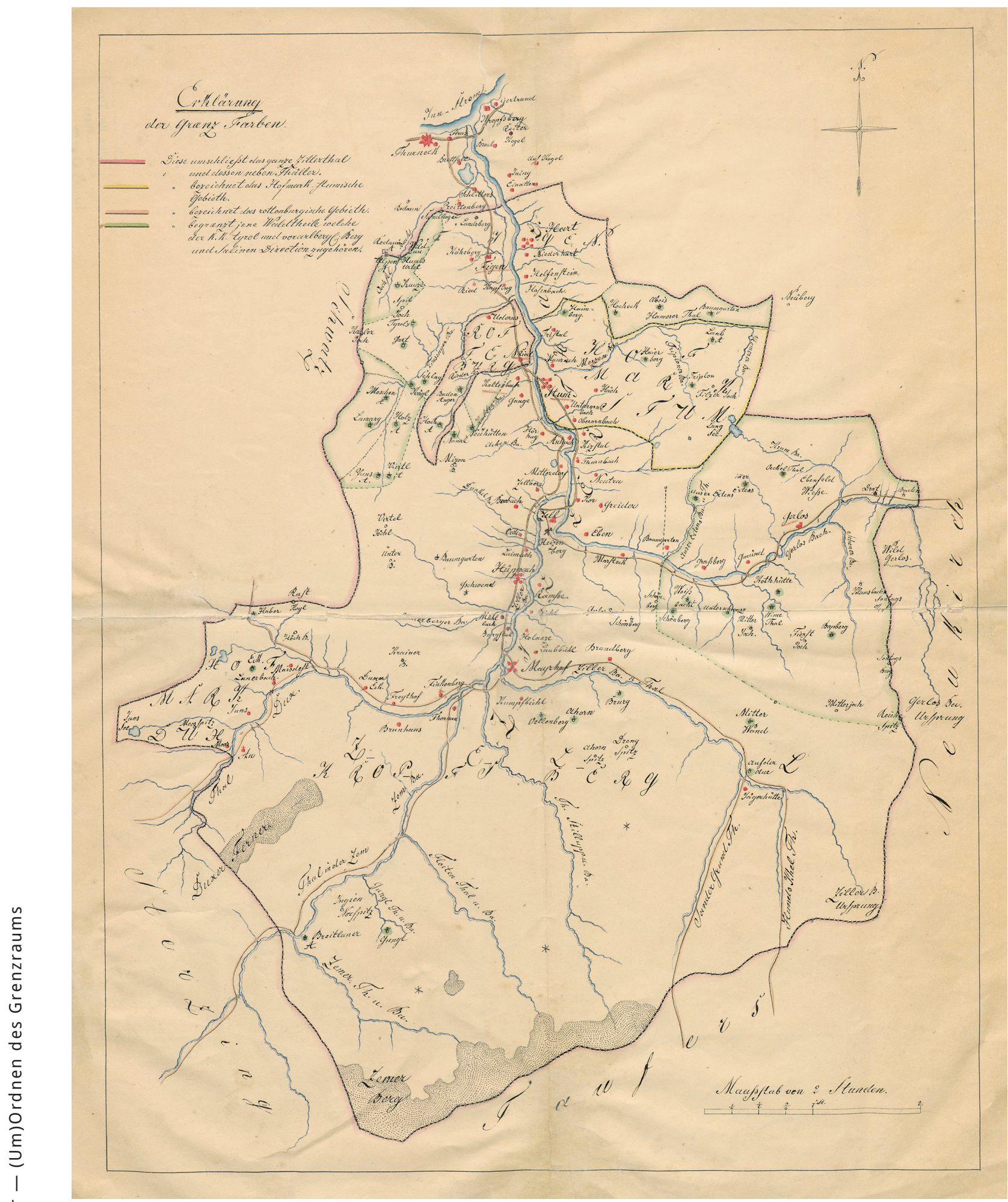

Abbildung 1: Karte des Zillertals, angefertigt durch den Bergwerksdirektor von Schwaz, Johann Anton von Erlach, 1766. In gelb und in einem helleren Rosa finden sich die Tiroler Gebietsanteile im Zillertal (die vom Landgericht Rattenberg verwaltete Hofmark Stumm und das Pfleggericht Rottenburg) eingezeichnet. In Richtung Talausgang liegt das Salzburger Pfleggericht Fügen, im hinteren Talbereich das Pfleggericht Zell (Kropfsberg) (Quelle: TLA, Karten und Pläne, Nr. 2867, Reproduktion: TLA). 
Nicht nur fehlten einige Markierungssteine oder waren aufgrund der Witterung nicht mehr richtig lesbar, manche waren offensichtlich auch falsch eingesetzt worden,

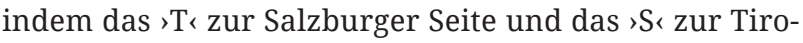
ler Seite wies. ${ }^{41}$ Bei zu großen Abständen zwischen den Markierungen regte der Pfleger an, dazwischen weitere anzubringen, um den genauen Verlauf klarer zu verdeutlichen, "um so mehr, als man von ersteren Markstein zum lezteren nicht einmal sehen kann «. ${ }^{42}$ Das Ideal war also offensichtlich ein Grenzmarkierungssystem, das es wie auf einer Karte ermöglichte, zumindest in der Vorstellung Linien von einem Punkt zum nächsten zu ziehen. Nicht alle Flurnamen waren seit der Einsetzung der Grenzsteine und dem Anbringen von weiteren Markierungen gleich geblieben, zum Teil hatte man 1711 offensichtlich auch alte aus den Quellen bekannte Namen einfach weiterverwendet, ohne im Zweifelsfall darauf zu achten, ob sich die Markierungen genau zuordnen ließen. Damit ergaben sich 1793 Unsicherheiten, wenn sich etwa die Bezeichnung einer Quelle - auch nach Rückfrage bei den angrenzenden Bauern - auf mehrere dort befindliche Quellen beziehen konnte. ${ }^{43}$ Konkrete Auswirkungen in der Raumnutzung zog dies nach sich, wenn Tiroler und Salzburger Untertanen die Grenzpunkte unterschiedlich interpretierten und demnach »sich die jenseitigen [...] Unterthanen nicht nur das Vieh der Salzburger Insassen ab, und ihres aufzutreiben, sondern sich sogar auch einen Holzschlag [...] zu machen erlaubten «. ${ }^{4}$ Trotz deutlich ersichtlichen Grenzverlaufs gaben die Bauern an einigen Stellen an, dass sie für Weidegründe, die auf der einen Seite der Grenze lagen, die Abgaben an die Herrschaft auf der anderen Seite entrichteten. ${ }^{45}$ Als eine Art `Niemandsland ‘ oder 'Jedermannsland` präsentierte sich der Grenzraum, wenn es etwa hieß, dass Quellen zum Tränken des Viehs von beiden Seiten genutzt wurden. ${ }^{46}$ Schließlich fand die Grenzbegehungskommission Markierungen, die sie nicht zuordnen konnte, und vermutete, dass es sich um ältere, nicht mehr gültige Waldmarkierungssteine handle ${ }^{47}$ oder sich überhaupt keine Angabe machen ließ, wie etwa über ein in einer "kleinen Kluft [...] im Felsen eingehauenes Kreuz [...], davon bisher noch kein Gränzprotokoll Meldung macht, und die Anwesenden hierüber eben keinen Aufschluß angeben konnten . $^{48}$

Damit hatten die Grenzbegehungen nicht die erhoffte exakte Klarheit der Grenzen gebracht. Je genauer man an bestimmten Abschnitten hinschaute, desto mehr wurde die vermeintliche Trennlinie wieder zu einem breiteren, nicht klar definierbaren Band, das Deutungsspielraum aufwies und zugleich in der Annäherung an die gewünschte Eindeutigkeit viel an Kommunikation erforderte, ohne sie letztlich exakt herstellen zu können. Offensichtlich war also das Wissen um den exakten Grenzverlauf nicht unbedingt notwendig, um den Raum trotzdem nutzen zu können. Grenzen konnten vage bleiben, solange das Raumhandeln in den eingeübten Bahnen ungestört blieb. Daraus lässt sich vorsichtig der Schluss ziehen, dass das Raumbild, verknüpft mit dem jeweiligen Raumwissen, an den Rändern ausfranste, nämlich dort, wo es an Bedeutung für das unmittelbare Handeln im Raum verlor. Genaue Raumkonturen >im Kopf`zu haben, scheint demnach nicht so wichtig gewesen zu sein.

Das belegt auch ein kurzer Blick auf einen ganz konkreten Grenzabschnitt, der zugleich zur nächsten Annäherung überleitet, nämlich zur Frage, ob Grenzmarkierungen für die Bevölkerung überhaupt eine Rolle spielten - angesichts des erwähnten Umstandes, dass sie diese Grenzen in ihrem Raumhandeln kontinuierlich überschritt. Der sogenannte `Sommerlange Zaun`stellte eine jener Markierungen dar, die sich schon im Landrecht des 15. Jahrhunderts erwähnt finden. Er verlief zwischen dem Salzburger Pfleggericht Fügen und dem Tiroler Landgericht Rattenberg vom Talboden in die Höhe und grenzte Felder voneinander ab. 1679 war eine Unsicherheit über den genauen Verlauf dieses Zauns entstanden. Etwa dreißig Jahre zuvor hatte ein Bauer im Landgericht Rattenberg am unteren Ende des `Sommerlangen Zauns` das angrenzende Gut auf der anderen Seite des Zauns, also im Pfleggericht Fügen, gekauft. In der Folge baute er das Stück Zaun, das nun mitten durch seine Besitzungen verlief, ab und errichtete ihn um seine Felder herum. Das rief den Protest der Nachbarschaft auf den Plan: Er könne diese alte Landmarkierung nicht einfach entfernen und verändern. Dies hatte also offensichtlich einen nicht hinnehmbaren Eingriff in das Raumbild dargestellt, der die Orientierung verändert hätte. So musste er den Zaun auch innerhalb eines Jahres wieder in seiner alten Form herstellen. Als dies 1679 zum Thema wurde, kam es vonseiten der Herrschaftsvertreter zu einer groß angelegten Rekonstruktion des ursprünglichen Verlaufs, um zu überprüfen, ob der jetzige 
Zaun noch mit der alten Grenzlinie übereinstimmte. Personen, die in an den Zaun grenzenden Gütern geboren waren oder dort gelebt hatten, wurden befragt, man fertigte genaue Situationsskizzen an, und von den Vertretern beider Herrschaften wurde ein "Augenschein" vorgenommen. ${ }^{49}$ Bei den Befragungen stellte sich heraus, dass die angrenzenden Bauern immer nur über den für sie relevanten Abschnitt des Zaunverlaufs Auskunft geben konnten. So beschrieb etwa der 77-jährige Martin Hochmuet den Zaun im Tal sehr genau, wie »weith oder hoch « dieser jedoch verlaufe, könne er nicht sagen, denn "auf alle Hech, alwo sich der Somerlange Zaun Endte«, sei er "Niemahls hinauf khomen «. ${ }^{50}$

Wenn es also - so wie hier, wo die Gerichtsgrenze mit Grundstücksgrenzen identisch war - um konkrete Grenzabschnitte ging, deren Veränderung auch einen Einschnitt in das Raumhandeln nach sich zog, dann stellten Grenzen nicht mehr nur eine Sache von Bedeutung für die Herrschaftsbeamten dar, sondern - in diesen Beispielen - auch für die bäuerlichen Grundbesitzer. Ein ähnlicher Befund zeigt sich, wenn Herrschaftsvertreter in der zweiten Hälfte des 18. Jahrhunderts bei der versuchten Klärung der Hoheitsrechte zugunsten des eigenen Territoriums stärker in das Raumhandeln der Talbewohner und Talbewohnerinnen eingriffen. Dabei lassen sich Unterschiede zwischen dem 18. und 19. Jahrhundert erkennen. Zunächst wird der Versuch der lokalen Herrschaftsbeamten geschildert, durch die Umleitung von Wegen Konflikte mit dem Gericht des benachbarten Territoriums auszutragen. Im beginnenden 19. Jahrhundert geht es danach um eine durchgeführte Verlegung von Herrschafts- und zugleich Diözesan- und Pfarrgrenzen.

\section{Verwalten des Raumhandelns durch (Um)Leiten von Wegen}

In der gemeinsamen Beteiligung aller Akteure und Akteurinnen an der Raumkonstruktion kam Herrschaftsvertretern die Macht zu, durch Verordnungen gewisse Wege zu sperren und in andere Richtungen zu leiten, allerdings, wie erwähnt, nicht ohne Auftrag oder Legitimation durch die übergeordnete Behörde. Dieses Vorgehen begann sich ab der Mitte des 18. Jahrhunderts zu intensivieren. Dahinter lässt sich ein erstes Bemühen die wirtschaftlichen Prozesse auf das eigene Territorium allein zu beziehen. Der Wirtschaftsraum des gesamten Zillertals war auf Tirol ausgerichtet. So zählten Innsbruck und die anderen kleineren Städte des Inntals zu den größten Abnehmern der Zillertaler Haupterzeugnisse Schmalz und Käse. Im Salzburger Gerichtsort Zell gab es jährlich Viehmärkte, die auch von Tirolern frequentiert wurden. In das vordere Salzburger Gericht Fügen wurde Bier aus dem tirolischen Jenbach eingeführt, die Salzburger ließen wiederum ihr Korn in Tiroler Mühlen mahlen, und die Wirtshäuser beider Herrschaftsgebiete wurden sowohl von salzburgischen als auch von tirolischen Untertanen besucht. ${ }^{51}$ Diese wirtschaftlichen Beziehungen gingen mit einem selbstverständlichen Überschreiten der Grenzen in beide Richtungen einher.

Die Initiative zur Übereinstimmung des Wirtschaftsraums mit den territorialen Grenzen ging um 1750 nicht vom Herrschaftszentrum aus, etwa im Sinne einer merkantilistischen Politik, sondern sie wurde vielmehr von den lokalen Herrschaftsvertretern strategisch in der gegenseitigen Konkurrenz eingesetzt. Diese als abrupt wahrgenommenen Eingriffe in die relationale Ordnung des Raums bewirkten in der Folge Reaktionen unterschiedlicher Akteure, mit dem Ziel, den Raum wieder szurückzuordnen`. An einem kleinen Stück Grenze, das durch Übertrittsverbote zusätzlich mit Bedeutung aufgeladen wurde, soll das Ineinandergreifen dieser Akteure gezeigt werden, die - jeweils ihre eigenen Interessen verfolgend - Allianzen bildeten, sich je nach Kontext entsprechender Argumentationsstrategien bedienten und dabei stets den Blick auf den Raum gerichtet hatten, denn es ging um nichts weniger als ihre Raumkonstruktion, die in Unordnung geraten war. Aufgrund unterschiedlicher Idealvorstellungen, wie sie den durch Handlungsoptionen strukturierten Raum geordnet haben wollten, folgten sie anderen Blickrichtungen und benutzten andere Argumentationslinien.

Als Bezugsstruktur dient eine erste grobe Unterscheidung nach den Ebenen der Talbevölkerung, der lokalen Herrschaftsvertreter und der Regierungsstellen im Herrschaftszentrum. Insbesondere in Hinblick auf die Bevölkerung ist nach der Zugehörigkeit zu sozialen Gruppen, nach Möglichkeiten der Artikulation von Beschwerden und Anliegen zu differenzieren. In den Quellen sichtbar werden befragte Einzelpersonen und Personenverbände, wie etwa die Bäcker oder die Wirte eines Ortes, die 
gemeinsame Bittschriften verfassten. Als Vertreter ihrer Herrschaften standen die Pfleger der beiden Salzburger Gerichte Fügen und Zell dem Tiroler Pfleger des Gerichts Rottenburg links des Zillers, das nominell zwar ein Patrimonialgericht darstellte, aber direkt der Repräsentation und Kammer beziehungsweise dem Gubernium in Innsbruck unterstand, und dem Tiroler Landrichter von Rattenberg rechts des Zillers gegenüber. In gleicher Weise wandten sich die salzburgischen Pfleger an den Hofrat in Salzburg oder direkt an den Erzbischof, um jedoch die Antwort stets vom Hofrat zu erhalten. Zwischen den lokalen Herrschaftsvertretern der beiden Territorien bestanden allerdings Rangunterschiede, die im persönlichen Verhältnis zumindest in manchen Konstellationen eine Rolle gespielt zu haben scheinen. Die Position der Salzburger Pfleger entsprach eher den Tiroler Kreishauptleuten, indem sie meist in gleicher Weise adelig und nominell zugleich Mitglied des Hofrats waren wie die Kreishauptleute nominell Gubernialräte. Die genannten Regierungsorgane im Zentrum traten ihrerseits miteinander in Kommunikation. Auch diese Behörden würden sich noch weiter differenzieren lassen - durch Hinzuziehung entsprechender Sitzungsprotokolle und Untersuchung der Entscheidungen in Hinblick auf den Anteil der einzelnen Räte sowie des jeweiligen Fürsten. Dies wird aber in den folgenden Überlegungen nur gestreift, da der Fokus auf dem lokalen Agieren und Argumentieren im und mit dem Raum liegt, damit also die Perspektive des Grenzraums eingenommen wird, wo die Entscheidungen des Herrschaftszentrums als verbindliche Einheitsmeinung wahrgenommen wurden, deren Zustandekommen vom lokalen Standpunkt aus nicht oder kaum nachvollziehbar war..$^{52}$

Zunächst zum konkreten Ort des Geschehens: Das salzburgische Dorf - und Sitz des gleichnamigen Gerichts wie auch Dekanats - Fügen gehörte, weil links des Zillers gelegen, zur Diözese Brixen. Zugleich unterstand der Pfarrhof mit seinen Grundstücken und Nebengebäuden der Tiroler Gerichtsbarkeit, bildete also innerhalb der Salzburger Landeshoheit eine kleine Tiroler Enklave. Zum Dorf hin war dieser >Widumsbezirk ‘ durch eine Mauer abgegrenzt. Wahrnehmbar konnte die unterschiedliche Jurisdiktion beispielsweise dann werden, wenn der Dechant sich bei weltlichen Anordnungen von Seiten Salzburgs auf seine Zuständigkeit nach Tirol berief und dadurch Salzburger Direktiven nicht umsetzen musste. So hatte er etwa in den 1780er Jahren die Möglichkeit, weiterhin Gottesdienste an aufgehobenen Feiertagen zu zelebrieren, obwohl die Salzburger Untertanen daran nicht teilnehmen durften. Auf diese Weise konnte auch der Dechant Druck und Sog auf die Bevölkerung ausüben, eingeübte Wege zu bestimmten Zeiten zu beschreiten, die nun jedoch durch die Landesherrschaft untersagt worden waren. Damit setzte er sich allerdings starker Kritik und Legitimierungsnot gegenüber dem salzburgischen Pfleger aus. ${ }^{53}$

1750 war ein an der Mauer liegendes Nebengebäude des Pfarrhofs - der sogenannte 'Widumkeller - ausgebaut worden und hatte von der Tiroler Regierung eine Ausschankerlaubnis für Bier und Branntwein verliehen bekommen. Rückblickend im Austausch der Argumente wurde diese Maßsnahme vordergründig damit gerechtfertigt, dass die Tiroler Untertanen im Zillertal, die zum Gottesdienst nach Fügen kamen und "sonderheitlich zu Winterszeiten ohne einige Labung nicht wohl nach Haus gehen kennen, nicht genöthigt seyen «, ihr Geld zum Nachteil des kaiserlich-königlichen Umgelds in ausländischen Wirtshäusern zu lassen. Doch in einem Nebensatz war auch die Rede davon, dass kurz davor der Salzburger Pfleger die Einfuhr des Biers aus dem Tiroler Ort Jenbach verboten hatte. ${ }^{54}$ Auch ein Teil der Bevölkerung in Fügen nahm diese neue Wirtstaverne offensichtlich gut an, die Menschen lenkten ihre Wege dorthin, ungeachtet des Umstands, dass sie auf Tiroler Boden lag.

Daneben lassen sich jedoch auch Gegenstimmen ausmachen, die sich jeweils an verschiedenen Aspekten der neuen räumlichen Anordnung stießen und diese revidieren wollten. Der 74-jährige Barthlme Gwerchner gab 1757 an, dass "jenes Heißl, welches vorhin der sogenannte Widumkeller war, aufgeführt und sowohl der Länge als Breite nach fast um ein Klafter unmittelbar auf Salzburg. Grund herausgefahren « worden sei. Er habe gemeinsam mit anderen Nachbarn mehrmals dagegen protestiert, weil aufgrund der Dachrinne, die nun über die Mauer herausragte, die Straße seither den ganzen Sommer hindurch nass und rutschig, im Winter aber eisig und damit für Fuhrwerke und Pferde schädlich und gefährlich sei. Zudem, so unterstrich er noch die durch die Veränderung entstandenen Nachteile, sei den benachbarten Häusern durch die Höhe des Gebäudes »das Liecht umb ein mörkliches, und den Uhrschlag zu hören benohmen worden . ${ }^{55}$ Andere - der Metzger Veith Rainer 
und der Krämer Veith Haydacher - unterstützten diese Aussage, auch wenn sie das Ausmaß, bis zu dem sich das Haus nun über die Grenze hinweg auf salzburgischen Boden erstreckte, nicht genau angeben konnten. ${ }^{56}$

Etwa zur gleichen Zeit wandten sich die "Wirte und Bierzapfler« im Gericht Fügen an den Erzbischof von Salzburg und bezogen sich darauf, dass sie schon öfter in den letzten Jahren darum gebeten hatten, in Bezug auf dieses aus ihrer Sicht widerrechtlich von Tirol mit einer Ausschankerlaubnis versehene Haus »eine unmaßgebigste Abänderung zu machen, [...] als uns dieser Ausschank [...] zu unser aller gresten Schaden " gereiche. Dieser Schaden sei "um so viel beträchtlicher [...], weil die Salzburg. Untertanen und ledige Bauern Pursch wegen in diesem Zaumb und Zigglosen Ort zu allen Excessen habenden Freiheit hausen«. Da es auf Tiroler Boden liege, stehe dem Salzburger Pfleger dort kein Zugriffsrecht zu. Das Tiroler Gericht hingegen würde "alles leichterdingen « geschehen lassen. Während also »in unseren Schankhäusern den zechenden Personen von dem Visitierenden« amtlicherseits befohlen worden sei, nach Hause zu gehen, könnten sie sich in diesem Haus "nach beliebender Länge der Zeit« aufhalten. Unterstützt wurde das Argument mit dem Hinweis auf den Schaden, der durch das entgehende Umgeld »dem hochfürst. Cammerale selbst» geschehe, und schließlich noch damit untermauert, dass diese Wirtstaverne ihre Befugnisse überschreite, indem sie zu »mehrern Schaden und völligen Ruin so gar Wein" ausschenke und warme Speisen anbiete. ${ }^{57}$

Bereits etwas früher - 1753 - hatte der Dechant selbst seine Bedenken gegen diesen Ausschank geäußert. Seine Argumente lagen in der geringen Entfernung des Hauses zu seinem "negst anstossenten Heystadl, und Stallungen «, was durch die "aus- und eingehente[n] Betrunckhenen Tabackhgehern « zu einer erhöhten Feuergefahr führen würde. Zudem - so merkte auch er an sei »dises Gewerb ausser aller Aufsicht der welltichen Obrigkheit gesetzt, ja sozusagen ein Schluf Winckhl der Polliceyordnung der Fügner.en Gerichts Obrigkheit«. Schließlich führte er noch an, dass der Bier- und Branntweinausschank auch an Sonn- und Feiertagen zur Zeit des Gottesdienstes stattfinde und somit die Leute vom Besuch der Messe abhalte. ${ }^{58}$

Diese Einwände standen in unmittelbarem Zusammenhang mit dem gleich noch zu schildernden Vorgehen hatte sich der Pfleger mit seinem Oberschreiber und zwei Beisitzern extra in dessen Wohnung begeben, da dieser bettlägerig war. Die Wirte und Bierzapfler konnten sich in ihrem Schreiben genau auf alle in der Sache bereits ergangenen Dokumente beziehen, was auf eine Abstimmung mit dem Pfleger schließen lässt. Schließlich zeigt auch der Hinweis des Dechants, der seine erste Beschwerde bereits 1751, also kurz nach der Errichtung der Wirtstaverne, erstmals formuliert hatte, auf den auch aus seiner Sicht fehlenden Durchgriff des Pflegers von Fügen, dass die beiden in dieser Sache die gleichen Interessen vertraten. Allerdings lassen sich trotz der in erster Linie vom Pfleger ausgehenden Initiative in den drei Blickrichtungen jeweils die eigenen Raumvorstellungen der handelnden Personen erkennen, die durch die Veränderung in persönlicher, wirtschaftlicher und seelsorgerischer Hinsicht beeinträchtigt wurden.

Der Pfleger von Fügen, Carl Leopold Lürzer, hatte ähnliche Argumente ins Treffen geführt und im November 1756 ein Verbot für salzburgische Untertanen erreicht, das Tiroler »Bier- und Brand Wein-Häußl hinkünfftig zu frequentirn, und darin zu zechen ". ${ }^{59}$ Doch fügte er in seiner Begründung weitere Aspekte hinzu, lag in seiner Verwaltungstätigkeit ja auch die Aufgabe, auf die Wahrung der Landeshoheit zu achten. Sein Raumkonzept und seine Blickrichtung erweiterten sich damit auf die aus seiner Sicht permanent drohende Verletzung der Grenzen durch die Herrschaftsvertreter des Nachbarterritoriums. Mit dem Abschneiden des Wegs für die Salzburger Untertanen in die Tiroler Wirtstaverne begab er sich in eine direkte Konfrontation mit dem Pfleger des Tiroler Gerichts Rottenburg, Michael Ignaz von Löffler.

Angelpunkt der Argumentation war stets die Frage, welches Vorgehen eine Neuerung darstellte und damit nicht legitim war. Löffler schrieb von »Befremden«, mit dem er den Schritt seines Fügner Kollegen wahrgenommen habe. Er bestritt der Salzburger Herrschaft das Recht, ein solches Verbot auszusprechen, das in das Tiroler Territorium hinein Auswirkungen nach sich ziehe. Zudem sei die angesetzte Strafe dafür unverhältnismäßig: Nicht nur würden Zuwiderhandelnde mit zehn Gulden bestraft, sondern im Wiederholungsfall sogar mit Schanzarbeit in Salzburg bedroht. Im Gegensatz dazu habe man Tiroler Untertanen den freien Zugang zu Salzburger Wirtshäusern noch nie verboten, sondern "beedseithigen ohnedeme untereinander vermischten 
Unterthanen nach alter Observanz mutuelle Freyheit“ zugestanden. Damit stelle das Salzburger Vorgehen aus der Perspektive Tirols eine Neuerung und ein unbefugtes sowie "unnachbarliches Betragen « dar, das "zu vilen Weitherungen die Thür erröffnen « könne. ${ }^{60}$ Lürzer wiederum sprach dem Tiroler Pfleger das Recht ab, sich über ein Verbot befremdet zu zeigen, das sich nur auf Salzburger Untertanen beziehe, besonders wenn derlei Verordnungen auf »Abstöllung übler Sitten und zeithero getrübenen Ärgernussen heylsamb abzihlen«. Dies sei daher keineswegs eine Neuerung. ${ }^{61}$ Stattdessen, so Lürzer später in seinem Rechtfertigungsschreiben an den Erzbischof, als sich die Repräsentation und Kammer direkt an Salzburg gewandt hatte und der Hofrat vom Pfleger in Fügen nun Aufklärung über die Vorgänge forderte, habe die Errichtung eines Schankgewerbes im Pfarrhof in Fügen und somit mitten im Salzburger Gebiet eine unrechtmäßige Neuerung dargestellt, da sich weder in den Verträgen noch im Verlauf der letzten zweieinhalb Jahrhunderte in den Quellen Hinweise fänden, die ein solches Gewerbe vorsahen. Als Beweis und zusätzliche Unterstützung seines Vorgehens schickte er die oben beschriebenen Aussagen und Berichte der Augenzeugen, der Wirte und des Dechants mit. ${ }^{62}$

Die Austragung des Konflikts war in allen Phasen eng mit dem Agieren im Raum verknüpft. Das Verlesen der Salzburger Verordnung hatte öffentlich in Fügen und einem Nachbarort stattgefunden. Ob der Oberschreiber dabei das Strafausmaß tatsächlich so, wie vom Rottenburger Pfleger wahrgenommen und festgehalten, verkündet hatte, muss dahingestellt bleiben. Die Verordnung selbst hatte keine Bestimmung über das Strafausmaß enthalten. Jedenfalls stritt Lürzer dies ab. ${ }^{63}$ Löffler drückte die Hoffnung aus, dass sein Gegenüber dieses Verbot sofort wieder öffentlich widerrufen werde, ansonsten sei er gezwungen, die Sache bei seiner vorgesetzten Behörde anzubringen. ${ }^{64}$ Daraufhin ließs Lürzer die Verordnung nochmals öffentlich verlesen - mit Angabe einer geringeren Strafe als der zuvor genannten. Das gab schließlich für Löffler den Ausschlag, sich an die Repräsentation und Kammer in Innsbruck zu wenden. ${ }^{65}$ Angekündigt hatte er zudem zuvor, dass es in diesem Fall dann keineswegs an Gelegenheiten fehlen dürfte, den Tiroler Untertanen in umgekehrter Weise den Besuch von Salzburger Wirtshäusern zu verbieten - bei doppelter Strafe. Darüber hinaus könne man auch den
Zugang zu den Zeller Viehmärkten, die dem Vernehmen nach immer mehr Ertrag brächten, für Tiroler Untertanen sperren. ${ }^{66}$ Diese Drohungen, so die Antwort Lürzers darauf, würden bei ihm "geringes Augenmerk" finden, weil dies in keinem Verhältnis zur Salzburger Anordnung stehe. Wenn die Angelegenheit an die höheren Behörden gehe, so vertraue er darauf, dass die gerechte Sache in seinem Sinn ausgehe. ${ }^{67}$

Als der Tiroler Pfleger 1761 im Verlauf der weiteren Auseinandersetzung das angedrohte Verbot für die Tiroler Untertanen, Salzburger Wirtshäuser zu besuchen, tatsächlich erwirken konnte, spielte wiederum dessen öffentliche Verkündung als ein Ereignis, auf dessen Platzierung im Raum ganz genau geachtet wurde, eine wesentliche Rolle. Der Salzburger Pfleger von Zell - etwas weiter entfernt vom Ort des Geschehens hinten im Tal - hatte das Gerücht vernommen, dass sich am Sonntag nach dem Gottesdienst in Fügen ein Schreiber aus dem Fenster des umstrittenen Hauses gelehnt und verlesen habe, dass sich alle Tiroler Untertanen bei Strafe der Salzburger Wirtshäuser enthalten sollen. ${ }^{68}$ Mit diesem Hinauslehnen habe er sich in Salzburger Hoheitsgebiet begeben. Daher war die wahrgenommene Provokation offensichtlich so groß, dass sich der Zeller Pfleger, obwohl das ganze Geschehen gar nicht in seiner Zuständigkeit lag, mit dieser Information sofort an den Erzbischof in Salzburg wandte. Nach einem Bericht des Schreibers von Fügen klärte sich das Vorgehen allerdings auf - nicht in der umstrittenen Wirtstaverne, sondern in den Tiroler Orten des Zillertals und damit in "ungezweiflet " Rottenburger Jurisdiktion sei die Verordnung publiziert worden. ${ }^{69}$

Ein kurzer Blick sei auch auf die bestimmenden Momente der zentralen Behörden geworfen, um sich deren Überlegungen zur Raumkonstruktion annähern zu können. Für den Hofrat in Salzburg zählte in dieser Auseinandersetzung als Argument zunächst vor allem das Bedenken, das bezüglich des Verhaltens der jungen ledigen Männer angeführt wurde, nämlich dass "weder ein verheyrather ansessiger Unterthan, noch einer von den ledigen Pursche « dort zechen dürfe. ${ }^{70}$ Ähnlich wie schon auf der Ebene der Herrschaftsvertreter im Tal sprach auch die Tiroler Repräsentation und Kammer Salzburg das Recht ab, ein solches Verbot zu erlassen. Die in diesem Haus »ausübende Excessen « seien nur "zur angeblichen Ursach vorgeschüzet worden «. ${ }^{71}$ Wenn es tatsächlich Exzesse gebe, dann seien diese ordnungsgemäß zu 
untersuchen und zu bestrafen, aber es könne nicht der Besuch eines Wirtshauses verboten werden.

Zugleich wurde auch ein anderes Verbot verhandelt, das hier mit hereingenommen wird, weil es zum einen in gleicher Weise Wege einzuschränken versuchte, zum anderen aber Licht auf die ideale Raumvorstellung der zentralen Behörden vor und um 1750 wirft. Die Repräsentation und Kammer warf dem Erzbischof auch vor, dass er nicht einfach das Kaufen von Brot bei Tiroler Bäckern verbieten könne. Es sei sehr bedenklich, den bisher ausgeübten "freyen Handl und Wandl« zu unterbrechen. Das würde zu einer Gewerbebeeinträchtigung der Tiroler Untertanen führen. ${ }^{72}$ Das Aufgreifen dieses Verbots verwies auf eine längere Praxis des Raumordnens zwischen den beiden Gerichten. Bereits 1742 hatte der Salzburger Pfleger ein Verbot erwirkt, dass die Salzburger Untertanen ihr Getreide nicht in Tiroler Mühlen mahlen lassen sollten. ${ }^{73}$ Auslöser war auch hier eine Veränderung im Raum gewesen - kurz hinter der Grenze auf Tiroler Boden war eine neue Mühle errichtet worden, die für viele Salzburger Untertanen näher lag als jene auf Salzburger Gebiet. ${ }^{74}$ Als jedoch 1748 zwei Fügner Bäckermeister das Ansuchen stellten, dass »denen Weibern das Brod von Thyroll herein zu tragen, und zuverkhauffen« verboten werde, kommunizierte der Salzburger Hofrat deutlich, dass, solange die Salzburger Bäcker ihr Brot auch in Tirol verkauften, das "jennseitige Hereinbringen desselben zu Beobachtung des reciproci gleichfahls gestattet « werden müsse. ${ }^{75}$ Tatsächlich war auch jenes Verbot von 1742 kaum beachtet worden. Zudem würden - in der Wahrnehmung Lürzers von 1757 nicht tolerierbar - sowohl Tiroler als auch Salzburger "Brodtrager und Weiber « zum Nachteil der hiesigen Bäcker in Tirol Brot kaufen und in Körben oder Schlitten auf Salzburger Gebiet bringen. Dort werde es öffentlich "und ihrzuweilen zu Truz deren Beckhen vor deren Läden verkauffet«. In seiner Rechtfertigung gegenüber dem Erzbischof wehrte er sich gegen das Argument des "reciprocums « und des freien Handels und Wandels, er sah darin eine Schieflage, die zu Ungunsten der Salzburger Bäcker ausfiel, und wollte sein Raumideal von geschlossenen Grenzen und damit der Demonstrierung der Landeshoheit durchsetzen. ${ }^{76}$

Der Salzburger Hofrat hingegen ließ zwar grundsätzlich erkennen, dass ihm die Wahrung der Landeshoheit wichtig war. Er trug seinen Pflegern auf, dies genau zu beobachten und darüber Bericht zu erstatten. Zugleich erfolgten jedoch stets Aufforderungen, ein gutes nachbarliches Einvernehmen zu wahren. In den 1750er Jahren ließ sich somit noch nicht nachweisen, dass das landesfürstliche Bestreben in Richtung eines abgeschlossenen Staatsgebietes ging, das jegliche wirtschaftliche Abhängigkeit über die Grenzen oder jegliche Einflüsse eines anderen Territoriums beseitigen wollte. ${ }^{77}$ Auch 1773 lautete die Anordnung des Hofrats hinsichtlich eines anderen Falls ähnlich. Der Rottenburger Pfleger hatte zwei Händlern aus Tirol den Platz unter der Traufe der Wirtstaverne in Fügen, die, wie erwähnt, durch den Ausbau auf Salzburger Gebiet hinausreichte, zum Verkauf ihrer Waren vermietet. Damit demonstrierte er, dass er diesen Platz als Tiroler Boden betrachtete. Daraufhin hatte der Salzburger Pfleger die beiden Händler festnehmen lassen und ihre Waren beschlagnahmt. Trotz seines skizzierten Schreckensszenarios, dass dies einen Versuch vonseiten Tirols darstelle, immer mehr an Boden zu gewinnen und sich auszubreiten, ordnete der Hofrat an, dass die Waren zurückzugeben und die Händler ohne Strafe wieder zu entlassen seien. Es sei zwar wichtig, auf solche Übergriffe zu achten, aber für »dieses mall« könne man es bei einer Abmahnung belassen. ${ }^{78}$

Es scheint also offensichtlich, dass um die Mitte des 18. Jahrhunderts die Initiative zur aktiven Raum(um)ordnung mehr von den lokalen Herrschaftsvertretern als von den zentralen Behörden ausging. Wie sehr diese Auseinandersetzungen auf der Austragung von persönlichen Konflikten beruhen konnten, zeigt die zusätzliche Anzeige, die Löffler 1757 vor der Repräsentation und Kammer in Innsbruck vorbrachte, nämlich dass Lürzer sich zu Unrecht des Titels eines Kriminalrats bediene. ${ }^{79}$ Lürzer - vom Salzburger Hofrat damit konfrontiert stritt dies ab, nicht er habe diesen Titel verwendet, sondern er sei von seinem Oberschreiber in einem Dokument, das er ihm als »Charta Bianca« zurückgelassen habe, als er aufgrund anderer Verrichtungen abwesend gewesen sei, so bezeichnet worden. Zugleich nutzte er seinerseits die Gelegenheit, sich über das Verhalten und die Ambitionen des Tiroler Pflegers zu beschweren: Anmerkungen zu dessen Naturell seien notwendig, um dessen Vorwurf zu erklären. Dieser habe den Titel eines Kaiserlich-Königlichen Pflegers angestrebt, aber nicht erreicht, woraus sein "gehässiges « Betragen und der Neid auf seinen, des Salzburger Pflegers, Rang resultiere. ${ }^{80}$ 
Alle drei >Untersuchungsgruppen ‘ konstruierten ihren Raum entlang der jeweils vorgegebenen Möglichkeiten und Gewohnheiten selbst. Durch den Eingriff der lokalen Herrschaftsvertreter mittels der erwirkten Verordnungen, Wege zu sperren und umzuleiten, gerieten die Raumkonzeptionen sowohl der Talbevölkerung als auch des Herrschaftszentrums in Unordnung. Zumindest wurden diese infrage gestellt und damit zu einer Reaktion herausgefordert. Von der Bevölkerung als solcher lässt sich, wie erwähnt, in historischen Kontexten nie sprechen, weil die Quellen, wenn es sich nicht um Massenquellen handelt, selten repräsentative Erhebungen zulassen. Sichtbar wurden Reaktionen einzelner von den jeweiligen Veränderungen betroffener Personen, wie etwa der Anrainer des Pfarrhofs oder der Wirte und Bäcker von Fügen. Sie nutzten das Mittel von Eingaben und Bittschriften beim Erzbischof, um den Raum in ihrem Sinne wieder zurückzuordnen. Das Beispiel der Bäcker zeigte, dass die Initiative zu einer Raumveränderung auch von einer Bevölkerungsgruppe ausgehen konnte. Wie erfolgreich diese Bittschriften waren, hing eng mit den Raumkonzeptionen der anderen Beteiligten zusammen. Die Herrschaftsvertreter trugen über die Raumumordnung die Konkurrenz mit dem Vertreter des Nachbargerichts aus und bedienten sich offensichtlich dann der Raumumordnungsversuche einzelner Bevölkerungsgruppen, wenn diese in ihrem Sinn waren. Die Raumkonzeptionen des Herrschaftszentrums in Salzburg, das, wie erwähnt, nicht weiter aufgeschlüsselt wurde, sondern als Einheit von Hofrat und Erzbischof in Form von Nachfragen und Beschlüssen im Tal in Erscheinung trat, waren von den Raumumordnungen der Herrschaftsvertreter im Tal ebenfalls betroffen. Zwar war für die Eingriffe der Beamten die Zustimmung des Regierungszentrums notwendig, doch lag es in der Argumentation der Herrschaftsvertreter, diese als notwendig und unumgänglich darzustellen. Die Bewilligung der Besuchsverbote der Wirtstaverne auf Tiroler Grund scheint zunächst kaum als gravierender Eingriff wahrgenommen worden zu sein. Erst die Reaktionen des Nachbarterritoriums - in Gestalt dessen Herrschaftsvertreters und Regierungszentrums - zwangen zu einer umfassenderen Auseinandersetzung und handelnden Stellungnahme. Die Aufträge an die Herrschaftsbeamten im Tal, die nachbarliche Eintracht zu wahren und den gegenseiti- ein Raumbild des Regierungszentrums mit zu diesem Zeitpunkt noch offenen und durchlässigen, wenn auch aufmerksam beobachteten Grenzen zu.

Die Ausstattung mit Macht, den Raum aktiv umzuordnen, war zwischen den einzelnen Gruppen klar ersichtlich ungleich verteilt. Das führte allerdings slediglich zu einer unterschiedlichen Wahl der Mittel bei der Konstruktion des Raumes: Je weniger Eigenmacht, aktiv Eingriffe vorzunehmen, desto mehr musste mit dem Mittel der Anpassung gearbeitet werden. Umgekehrt war das Herrschaftszentrum auf die Beamten angewiesen, seine Vorstellung des Raums an Ort und Stelle umzusetzen, und beide Gruppen auf die Bevölkerung, die Anordnungen zu akzeptieren. Damit waren die Raumkonzeptionen aufs Engste miteinander verwoben.

\section{Neu gezogene Raumgrenzen nach 1800}

In den nachfolgenden Jahrzehnten begannen sich die Raumvorstellungen auch der zentralen Stellen zu verändern. Im Kontext der begonnenen Ausgleichsverhandlungen und des Versuchs, die eigenen Territorien zu arrondieren, scheinen die Konflikte und die Notwendigkeit, über den Raum, seine Grenzen und die jeweilige Landeshoheit zu kommunizieren, zugenommen zu haben. Dies war etwa der Fall beim neuen österreichischen Mautsystem der 1780er Jahre, das sich unmittelbar auch auf das Zillertal auswirkte. Zollschranken, die bei gleichzeitiger Abschaffung der Binnenzölle an die Territorialgrenzen gelegt werden sollten, stellten Eingriffe in das Raumhandeln dar und machten zugleich die Schwierigkeit offenkundig, bei territorial vermischten Gebieten den Zoll an der gewünschten Stelle zu platzieren. Im Fall des Zillertals mussten Tiroler immer auch Salzburger Gebiet überqueren, um an die äußerste Landesgrenze zu gelangen. Das führte beispielsweise zu Überlegungen vonseiten Österreichs, Teile des Nachbarterritoriums mauttechnisch in das eigene einzuschließen - ein Angebot, das der Salzburger Hofrat zwar aufgrund der damit verbundenen Vorteile ernsthaft diskutierte, aber wegen der befürchteten Gefährdung der Landeshoheit schließlich ablehnte. ${ }^{81}$

Die Notwendigkeit, nach dem Frieden von Campo Formio von 1797 die linksrheinischen Fürsten zu entschädigen, hatte zu den Verhandlungen in Rastatt und 
schließlich in Regensburg mit dem Ergebnis des Reichsdeputationshauptschlusses von 1803 geführt. Alle vorgebrachten Überlegungen, welche Gebiete aufgrund von Flächeninhalt, Bevölkerung und Umsatz als Entschädigung für welche Fürsten gelten konnten, waren aufgrund der immer genauer durchgeführten Vermessungen und Zählungen möglich geworden. 1803 kam es vor allem durch die Mediatisierung zu umfassenden politischterritorialen Umordnungen des Raums, die einzelne 'Staatsgebiete immer geschlossener werden ließen. Eine logische Fortsetzung dessen scheint der Artikel 15 im Frieden von Pressburg von 1805 gewesen zu sein. Darin verzichtete der Kaiser "von Deutschland und Österreich" für sich und seine Nachfahren auf alle Gerechtsame und Ansprüche im Gebiet der Könige von Bayern und Württemberg sowie des Kurfürsten von Baden. ${ }^{82}$ Das bedeutete für Kaiser Franz II./I. zum einen die Aufgabe der letzten Besitzungen in den vorderösterreichischen Ländern. Zum anderen berührte diese Frage auch den Grenzraum zwischen Tirol und Salzburg, denn durch den Friedensschluss hatten sich die territorialen Zuständigkeiten geändert - Tirol war mit Vorarlberg an Bayern gefallen, Salzburg und Berchtesgaden gehörten nun zu Österreich. Damit standen auch die beidseitigen terrritorialen Einschlüsse im Zillertal zur Diskussion. Die zentralen Behörden in Wien gingen in ihrer Argumentation davon aus, dass der bayerische Hofkommissär in Innsbruck sehr bald Befehl erhalten würde, die salzburgischen Enklaven in seinem Gebiet in Besitz zu nehmen. Sie wollten daher möglichst rasch die Verhältnisse klären und ihrerseits Gebiete, die sich als bayerische Einschlüsse in ihrem Territorium betrachten ließen, in ihr Land eingliedern. Dazu wurden in der ersten Jahreshälfte 1806 von Salzburg aus Kommissäre zur Klärung der Frage, auf welche Gebiete man dabei Anspruch erheben konnte und welche wahrscheinlich aufzugeben waren, in die Grenzgerichte geschickt. Ähnlich wie bei den Grenzbegehungen von 1793 und 1798 kam es dabei zu einem Übereinanderlegen und Vergleichen der Raumvorstellungen der Bevölkerung mit jenen der Herrschaftsvertreter. ${ }^{83}$

Auf dieser Basis nahmen in Österreich die Pläne konkrete Gestalt an, sich vermeintlich zustehendes Gebiet notfalls mithilfe von Militär - anzueignen. Dabei handelte es sich zunächst um eine herrschaftliche Inbesitznahme einiger Grenzgebiete, die als saktive Enklaven 1780er Jahre von Joseph II., Herrschafts- und Diözesangrenzen in Übereinstimmung zu bringen, wieder aufgegriffen worden. Den Bischöfen von Brixen, Passau, Freising und von Chiemsee wurde die Loslösung aus ihrer Unterordnung unter die Metropolie Salzburg angeboten und dafür von ihnen die Aufgabe ihrer Rechte in den Pfarreien und Vikariaten im Salzburger Hoheitsgebiet verlangt. Hier richtete sich der raumordnende Blick auf die `Einsprengsel der anderen Diözesen. Die Intention war damit dieselbe - es sollte ein einheitlicher Raum ohne Einfluss eines anderen Herrschers hergestellt werden. ${ }^{84}$ Im Ergebnis kam es zur tatsächlichen Abänderung der Grenzen - sowohl im weltlichen als auch im kirchlichen Bereich. Für die betroffene Bevölkerung stellte dies einen abrupten Einschnitt in ihre Raumkonzeption dar, indem diese Veränderungen Handlungsroutinen abschnitten und eine Neuorientierung notwendig machten. An einem wiederum kleinen Grenzabschnitt wird nun danach gefragt, wie sich die Bevölkerung diesen veränderten Raum aneignete und ihre Raumperspektiven anpasste.

Auf der linken Seite des Zillers im Tiroler Gericht Rottenburg, an der Grenze zum Salzburger Gericht Fügen, befand sich der kleine Weiler Finsing, dessen Häuser entlang eines Bachs gereiht vor allem Gewerbe und Kleinhandel ausübten - es gab Müller, Gerber, Schmiede, Metzger, Bäcker und Krämer, aber auch Knappen wegen der nahe gelegenen Eisenbergwerke (Abb. 2). Diesen Gebietsstreifen erklärte der Fügner Pfleger aufgrund einer Anordnung der Landesregierung in Salzburg 1807 als nunmehr salzburgisches Hoheitsgebiet. Zugleich wurden Anpassungen der Diözesangrenze vorgenommen - die Pfarrei Fügen und die Kuratie Hippach wurden aus der Diözese Brixen gelöst und Salzburg zugeordnet. Damit waren aus der Perspektive Salzburgs die in den Austauschverhandlungen immer wieder als die eigenartigsten Vermischungen bezeichneten Einschlüsse beseitigt. Der herrschaftlich in Besitz genommene Weiler Finsing war bis dahin ein Teil der Lokalkaplanei des Tiroler Dorfs Uderns gewesen. Dem neuen Übereinstimmungsideal von Herrschafts- und Diözesangrenzen entsprechend war auch dies zu ändern, Finsing wurde daher nach Fügen umgepfarrt.

Die betroffenen Personen mussten damit nicht nur den Weg zum sonn- oder feiertäglichen Gottesdienst abändern, sondern waren auch für Taufen und Begräbnisse 


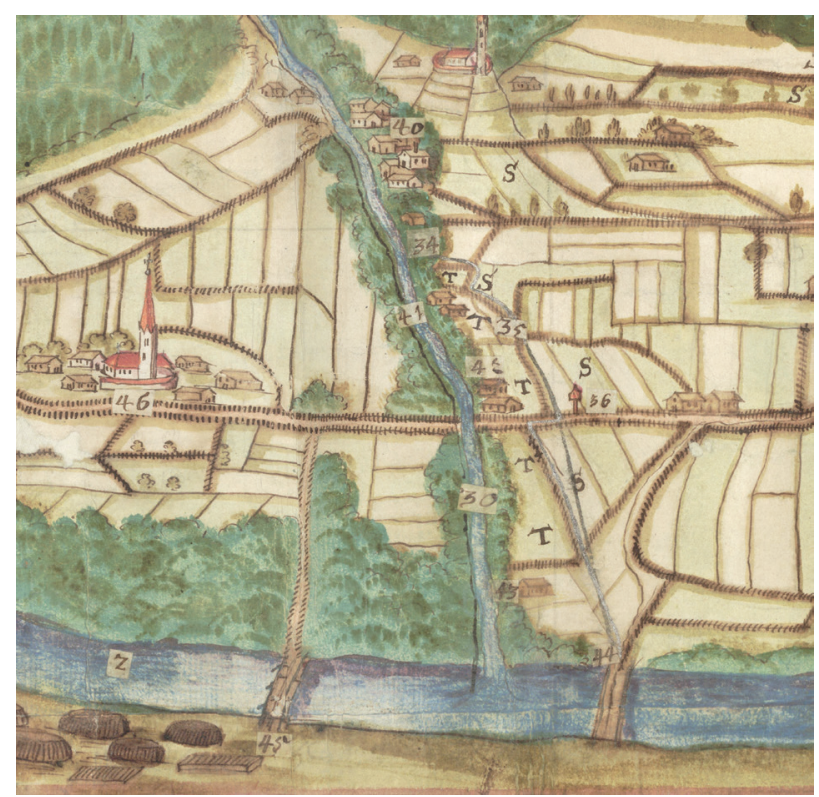

Abbildung 2: Der Weiler Finsing auf der Karte des Bergwerkdirektors von Schwaz Jeremias RambImayr, 1690. Die Gebäude am Finsingsbach sind hier - mit IT gekennzeichnet - noch Tirol zugeordnet, liegen jedoch sehr nahe der mit isı gekennzeichneten Grenze zu Salzburg. Nr. 46 verweist auf das Dorf Uderns im Tiroler Pfleggericht Rottenburg (Quelle: TLA, Karten und Pläne, Nr. 366, Reproduktion: TLA).

auf neue Orte verwiesen, auf die, insbesondere im Fall von Friedhöfen, nicht - sofort - die gleiche emotionale Bindung übertragen werden konnte. Das Dilemma, in das die Bevölkerung dadurch geriet, lässt sich an einem Fall vom Frühjahr 1808 zeigen. Als der neunjährige Müllersohn Veit Pircher in Finsing starb, stellte sich für die Angehörigen die Frage, wo er beerdigt werden sollte. Später im Verhör darauf befragt, gaben die Mutter und der ältere Bruder, der das väterliche Gewerbe übernommen hatte, an, dass sie vom Kuraten in Uderns aufgefordert worden seien, den Sohn beziehungsweise Bruder wie gewohnt in Uderns zu begraben. Die Abholung durch den Tiroler Gerichtsboten habe schließlich den Ausschlag gegeben. Aber sie räumten auch ein, dass es vor allem der Mutter schwergefallen wäre, den Sohn an einem anderen Ort als dem gewohnten begraben zu lassen:

Da nun mein Mann und 8. Kinder ohnehin zu Uderns im Gottesacker liegen, so sah ich es nicht gern, daß daß es mein Wille war, daß das Kind hinkomme, wo der Vater läge. ${ }^{85}$

Auf fehlendes Raumwissen konnten sie sich nicht beziehen, denn die Umpfarrung hatte in feierlicher Form mit Ablegung eines Gelübdes der Betroffenen stattgefunden. ${ }^{86}$ Solange jedoch die Möglichkeit - unterstützt von den Herrschaftsvertretern des anderen Territoriums - noch offen stand, versuchten sie, an ihrem gewohnten Raumhandeln mit den vertrauten Bezugspunkten festzuhalten.

Auch die herrschaftliche Umordnung war als offizieller Akt vorbereitet worden. Die Hausbesitzer von Finsing waren zuvor informiert worden, dass sie nun nach Fügen zuständig seien. Sie bekamen den Auftrag, in das Pfleggerichtsgebäude in Fügen zu kommen, um dort ihre Besitz- und Lehensurkunden vorzulegen - und sich auf ihre neue Herrschaft vereidigen zu lassen. Diese Umordnung ging jedoch nicht ohne Protest des Tiroler Pflegers von Rottenburg vonstatten. Dieser sei, so der Bericht des Salzburger Pflegers nach Salzburg, am Abend zuvor von Haus zu Haus gegangen und habe die Urkunden eingesammelt, um deren Übergabe und Neuausfertigung durch das Salzburger Gericht zu verhindern. Damit trat für die Bevölkerung eine Unschärfe ein, wie der Raum in herrschaftlicher Sicht wahrzunehmen sei, wohin sie nun tatsächlich gehörten, was eine Neuorientierung erschwerte. Die betroffenen Personen folgten dem Befehl und erschienen am festgesetzten Tag in Fügen, allerdings ohne ihre Urkunden. Trotzdem wurde ihnen kundgemacht, dass sie "nun der k. k. Landeshoheit unterworfen « seien, und sie mussten geloben, "hinfüro keinem Befehle von Tyrol mehr Gehör zu geben und dahin keine Giebigkeit mehr zu entrichten $«{ }^{87}$

Die Finsinger hielten nicht nur am gewohnten Begräbnisort fest, sondern ließen auch ihre Kinder weiterhin in der Kirche von Uderns taufen, zumindest ein entsprechender Fall ist in den Akten dokumentiert. Dadurch scheint dem Pfleger in Fügen bewusst geworden zu sein, dass die Umorientierung der Bevölkerung nicht so leicht nur durch Befehl zu verordnen war. Zudem stellte sich heraus, dass auch die Bezahlung der Steuern nicht einfach von einem Gerichtsort auf den anderen übertragen werden konnte. Der Müller und Bäcker Peter Pircher gab im Verhör an, dass er bei der Einfuhr des Getreides vom entsprechend instruierten Tiroler Zöllner vor die Wahl gestellt worden sei, entweder für das Getreide Maut zu 
bezahlen, wie es Salzburger Untertanen tun mussten, oder sich durch seine Steuerentrichtung nach Tirol als Tiroler Untertan zu erweisen. Der Pfleger von Rottenburg habe zudem verlauten lassen, dass er und die anderen Gewerbetreibenden, wenn sie die Steuer nicht in Tirol bezahlten, nicht nur das eingeführte Getreide verzollen müssten, sondern sich auch darüber im Klaren sein sollten, dass im angrenzenden Uderns in Tirol "ein neuer Mühler - Bäcker - Krämer etc. aufgestellt würde«. Daher habe er sich entschlossen, »lieber an beede Herrschaften zu zahlen, als ganz zu Grund zu gehen ${ }^{88}$ Auch der zweite Bäcker und Müller in Finsing, Josef Widgschwentner, hatte sich nach einem Vergleich seines Absatzes in Tirol und in Salzburg - in den Tiroler Orten belieferte er fünf Wirte, im salzburgischen Zillertal jedoch nur einen dazu entschieden, die Steuer zusätzlich nach Tirol zu zahlen. ${ }^{89}$ Die Krämerin Dorothea Mayrin bestätigte die Aussagen der beiden Bäcker. Sie hätte die Steuer nicht bezahlt, wenn sie "nicht alle Sonntage und Feyertage zu Uderns bey der Kirche feil hätte, und man mir nicht gedroht hätte, daß ich dieses Recht verliehren und ein neuer Kramer in Uderns entstehen würde, wenn ich diese Steuer nicht erlegete ${ }^{90}$ Auch der Metzger Franz Rainer befürchtete, dass im Tiroler Nachbarort ein neuer Metzger bewilligt würde, was sein Gewerbe deutlich beeinträchtigen würde. Er habe sogar schon gehört, dass dort ein Metzger bereits Interesse dafür angemeldet habe. ${ }^{91}$ Der Nagelschmied Paul Reindl schilderte einen ähnlichen Sachverhalt. Er habe dem Tiroler Gerichtsboten die verlangte Steuer bezahlt, weil er sonst »keine Nägl nach Tyrol verkaufen oder einführen « dürfe. ${ }^{92}$ Dem anderen Nagelschmied Joseph Sandbichler habe man gedroht, ihm kein Holz mehr zukommen zu lassen:

\section{Zudem sind wir ja 5. Naglschmid auf einem kleinen Fleck [...] und, wenn wir die Steuer nach Tyrol zu bezahlen verweigern, auch von jenen Nägeln, die wir nach Hall und andern Orten in Tyrol lieferten, eine unerschwingliche Mauth zahlen müßten, so zwingt mich ja wohl die Noth, diese Steuer zu erlegen. ${ }^{93}$}

Für Georg Stell, einen Knappen im Eisenbergwerk Schwaz im Tiroler Unterinntal, hatten sich schließlich durch die Veränderung der Grenzen Wohn- und Arbeitsort auf zwei verschiedene Herrschaften verteilt. Ihm habe man gedroht, so seine Frau im Verhör, dass er "sein Brod als
Bergknapp verliehren würde«, wenn er die Steuer an Tirol nicht zahlte:

Da wir nur vom Gütl nicht das halbe Jahr zu essen hätten und die Kinder sammt uns ohne Brod wären, wenn mein Mann abgedanckt würde, zu dem wir auch Holz, Streu und Weyde nur vom Tyrolischen erhalten können, so entschlossen wir uns halt, und bezahlten die Steuer. ${ }^{94}$

Alle gaben an, dass sie deshalb trotzdem ihre Steuer auch nach Salzburg zahlen würden - in den Worten des Nagelschmieds Joseph Sandbichler: "Ich dachte mir, wenn ich die Steuer nach Fügen auch bezahle, so werden sie nichts dawider haben ${ }^{95}{ }^{95}$ Damit verweigerten sie sich einer eindeutigen Zuordnung und der von beiden Herrschaftsvertretern geforderten klaren Abgrenzung von Räumen. Zumindest in der Übergangsphase beschritten sie beide Wege, integrierten also die Ausrichtung hin zur neuen Landesherrschaft in ihr altes Raumbild und brauchten dieses somit nicht aufzugeben.

Der Pfleger von Fügen ermahnte nach den Verhören alle, in Zukunft diesen Aufforderungen zur Steuerzahlung vonseiten Tirols keinesfalls mehr nachzukommen. Doch waren sich die Salzburger Beamten des Dilemmas sehr bewusst. Es lasse sich nicht verkennen, schrieb der ins Zillertal abgeordnete Kommissär Trauner im Bericht an die Landesregierung, dass die Lage dieser Untertanen unter diesen Verhältnissen immer drückender werde. Ihre Verbindung mit Tirol sei

durch die natürliche Begränzung unabänderlich begründet und selbst der Besitz des ganzen Zillerthals mit allen seinen Enclaven kann die Fortdauer dieser Verbindung und des wechselseitigen freyen Handelsverkehrs nicht aufheben, ohne die einzelnen Unterthanen in ihrem Erwerbe empfindlich zu drücken und ohne selbst fürs ganze Thal aufs nachtheiligste zu wirken. ${ }^{96}$

$\mathrm{Zu}$ einer Lösung dieses Konflikts kam es vorerst nicht, und im Jahr darauf änderten sich durch den Krieg von 1809 die territorialen Verhältnisse wiederum. Mit dem Frieden von Schönbrunn im Oktober 1809 fiel auch Salzburg an Bayern, und damit befanden sich beide Teile des Zillertals erstmals unter einer gemeinsamen Landesherrschaft. 
In diesem geschilderten Fall ging die Initiative zu einer Raumumordnung nicht von den Herrschaftsbeamten im Tal, sondern vom Regierungszentrum aus. Auch dieses konnte Grenzen nicht einfach verlegen, sondern musste das in Richtung des Nachbarterritoriums argumentativ begründen können, indem es die Rechtmäßigkeit beispielsweise von Friedensverträgen, hier von jenem von Pressburg vom 26. Dezember 1805, ableitete. Die Arrondierung des Staatsgebiets durch die Auflösung von territorialen Enklaven war in der Folge der Kriege, Friedensschlüsse und Gebietsentschädigungen zu einem `Dauerthemar geworden. Dies war mit der bereits zur Mitte des 18. Jahrhunderts intensiver einsetzenden Erfassung der Territorien mittels Zählung der Untertanen, Erstellung von Katastern und Kartierungen Hand in Hand gegangen oder dadurch erst befördert worden. Damit lässt sich im Blick des Herrschaftszentrums auf den Raum in dieser Zeit ein deutlicher Unterschied zur Haltung von noch etwa 50 Jahre zuvor erkennen. Viel deutlicher trat nun die Idealvorstellung eines abgeschlossenen Territoriums zutage. Die Raumkonzeptionen der Herrschaftsvertreter und der betroffenen Bevölkerung im Zillertal hingegen änderten sich weniger offensichtlich. Die Salzburger Beamten führten die Befehle zur Umordnung aus, klärten die dadurch neu gewonnenen Untertanen darüber auf und verwalteten das neue Raumgefüge. Das zunehmende Abschließen der Herrschaftsgrenzen entsprach ihrem Raumhandeln der vorhergehenden Jahrzehnte. Die Tiroler Herrschaftsvertreter weigerten sich, diese neuen Raumkonstellationen zu akzeptieren, forderten die umgeordnete Bevölkerung des Weilers Finsing auf, die neuen Verhältnisse zu ignorieren, und brachten entsprechende Druckmittel ins Spiel. Im Abwägen der Bedingungen erweiterten die betroffenen Handwerker, Händler und Bergknappen ihr Raumhandeln und integrierten die notwendig gewordenen neuen Wege zur Salzburger Herrschaft, ohne die bisherige Raumkonzeption umfassend zu ändern. Konfrontiert mit dem Dilemma, in das die Finsinger durch die neue Situation geraten waren, ließ ein Salzburger Beamter schließlich noch einen relativierenden Einblick zu, der von der Schwierigkeit zeugt, selbst in einer Zeit, in der alles auf die Geschlossenheit des Staatsgebietes konzipiert war, die abstrakt gedachte abgerundete Raumvorstellung mit den Verhältnissen in der Praxis in Überein-

\section{Raum als notwendige Kategorie? Abschließende Überlegungen}

Die von den Herausgebern in der Einleitung angesprochene Frage, ob der Zusammenhang von Staat und Raum eine Selbstverständlichkeit darstelle, die nicht weiter zu untersuchen sei, oder ob es sich dabei um einen lohnenden Forschungsgegenstand handle, ${ }^{97}$ lässt sich hinsichtlich des vorliegenden Beitrags in leicht abgewandelter Form stellen: Hätte sich >die Geschichte Bezüge zum Raum in gleicher Weise erzählen lassen, vielleicht als Geschichte gegenseitiger Konkurrenz der Wirte und Bäcker, der Herrschaftsvertreter und der Landesfürsten, und hätte diese Erzählweise dieselben Ergebnisse gebracht? Führt die Kategorie ‘Raum` demnach zu einem Mehrwert?

Zunächst einmal stellte das durchgehende Thema jenes der Grenzen dar. Der Forschungsblick folgte jenem der Akteure auf eine in unterschiedlicher Art kommunizierte Linie im Raum, die je nach Zugehörigkeit zu einer Gruppe mit verschiedenen Bedeutungen versehen war. Für die Herrschaftsvertreter umfasste sie das ihnen zugeteilte zu verwaltende Gebiet; wo diese Linie zudem eine fremde Herrschaft abgrenzte, galt es, auf die Wahrung der Landeshoheit zu achten. Für Teile der Bevölkerung trat die Bedeutung von Grenzen dann hervor, wenn durch eine Veränderung ihre Handlungsoptionen zur Diskussion standen. Das Handeln im Raum, aufs Engste verbunden mit Raumwissen, also dem Wissen vom Handeln im Raum - wie, entlang welcher Wege, zu welchen Zielen - ist ein unabtrennbarer Bestandteil menschlichen Tuns. Damit wäre man wieder am Beginn des Fragens angelangt: Ist das Raumhandeln so selbstverständlich, dass es keiner Erwähnung lohnt?

Den aktuellen Ansätzen der Raumanalyse zufolge konstruiert jedes Individuum entlang bestimmter Parameter den Raum für sich, indem alle vorgefundenen Subjekte und Objekte in einer relationalen Ordnung verknüpft werden. Diese Konstruktionen werden innerhalb gleicher - sozialer, funktionaler - Gruppen geteilt, gemäß dieser Anordnungen prägen sich durch das entsprechende Handeln und Sprechen darüber Routinen aus, die zugleich Orientierung ermöglichen und Sicherheit verheißen. Wenn nun an einer Stelle - abrupt - in dieses Gefüge eingegriffen wird, etwa durch bauliches Überschreiten einer Grenze, Zugangsverbote oder ein neues Definieren 
des Raums durch die Verlegung von Grenzen, wirkt sich das auf die gesamte Raumkonstruktion aus und bringt sie in Unordnung. In der Folge kommt es zu Reaktionen, die darauf abzielen, die gewohnte Anordnung wieder herzustellen oder die Raumkonstruktion entsprechend anzupassen. Diese gesamte Raumwahrnehmung der Individuen oder der verschiedenen Gruppen in ihrer Bedeutung für die damit verknüpften Handlungsoptionen und Routinen im Forschungsblick zu haben, erweitert das Erklärungsspektrum für historisches Handeln. Dementsprechend würde sich auch die Zuschreibung von Bedeutung einzelner Vorkommnisse, also Veränderungen im Raum differenzierter und zugleich umfassender - bezogen auf die jeweilige Raumkonstruktion - gestalten.

Entlang der vorgegebenen Pole 'Staat‘, ’Raum ‘ und ,Verwaltung، ließ sich das Ineinandergreifen der staatlichen Ordnungsvorstellungen, deren Verwaltung und damit Lenkung der Untertanen beobachten. Die unterschiedlichen Raumvorstellungen - hier ansatzweise aus der Blickrichtung einzelner Bevölkerungsgruppen, der Herrschaftsvertreter und des Regierungszentrums betrachtet - unterlagen im Untersuchungszeitraum einigen Veränderungen. Am deutlichsten trat dies auf der Ebene der Landesfürsten und deren oberster Behörden hervor. Waren diese in der Mitte des 18. Jahrhunderts noch darum bemüht, im Zillertaler Grenzraum einen gegenseitigen offenen Austausch über die Herrschaftsgrenzen hinweg aufrechtzuerhalten, so gingen um und nach 1800 die Bemühungen dahin, das Territorium klar abzugrenzen und alle Vorgänge auf die eigene Hoheitsgewalt zu beziehen. Das entsprach den veränderten Staatsvorstellungen eines klar umrissenen Herrschaftsgebiets, das sich ohne als fremd definierte Einflüsse bis an die Grenzen regieren ließ, wie sie - im Forschungskontext mit den Begriffen >Territorialisierung ^ und `Durchstaatlichung bezeichnet - für viele europäische Staaten beschrieben worden sind. Am Beispiel des salzburgisch-tirolischen Grenzraums lässt sich die Entwicklung dieser sich verändernden Vorstellungen von `Staatlichkeit` sogar sozusagen schrittweise über diesen Zeitraum hinweg verfolgen, da im Hintergrund der hier geschilderten Konflikte stets auf der Ebene von Gesandten der beiden Territorien über Ausgleichsmöglichkeiten von Rechten und Gebiet verhandelt wurde und in diese Diskussion immer neue Elemente und Argumente aufgenommen wurden. Das Zi-
Verhandlers - Gottfried von Moll am Beginn der Ausführungen, in dem er seinem Unverständnis Ausdruck verlieh, wie es nur dazu kommen konnte, dass Rechte und Territorium so zersplittert seien, deutet das an. 1780 hatte sich die neue Idealvorstellung von 'Staatsraum ‘ offensichtlich bereits so weit durchgesetzt, dass die frühneuzeitlichen Strukturen nicht mehr verstanden oder als sinnvoll möglich erachtet wurden.

Die Herrschaftsbeamten im Tal stellten die vermittelnde Verwaltungsinstanz des Regierungszentrums zur Bevölkerung dar. Sie erwiesen sich dabei jedoch keineswegs als seinfach Ausführender herrschaftlicher Vorstellungen und Anordnungen. Die anfangs tendenziell offenere Raumkonstruktion des Herrschaftszentrums traf vielmehr auf eine eher misstrauische, die Grenzen hütende, abschließende Raumvorstellung der lokalen Beamten, die aus der ausgetragenen Konkurrenz mit dem Gegenüber im Nachbarterritorium verständlich wird. Die Herrschaftsträger `spielten` dabei das gesamte Spektrum ihrer Verwaltungsbefugnisse und -techniken aus. Mit geschickter Argumentation erwirkten sie von den vorgesetzten Behörden die Genehmigung für die Verwirklichung ihrer Raumvorstellungen, indem sie beispielsweise Wege der Untertanen in ihrem Sinne sperrten und umleiteten. Die üblichen mündlichen Verkündungen von Anordnungen ließen stets Spielraum zu einer leichten Abänderung der Inhalte offen, die bei Bedarf im Nachhinein auch abgestritten werden konnte. Selbst wenn die Konflikte bis zur Ebene der Tiroler Repräsentation und Kammer beziehungsweise des Guberniums sowie des Salzburger Hofrats vordrangen und in der Folge umfassender legitimiert werden mussten, waren es immer noch die Herrschaftsvertreter, die aufgrund ihrer Position als Autoritätspersonen bestätigende Aussagen von Untertanen leichter motivieren und einsammeln konnten. Zudem verfügten sie über den ıwertvollen Schatz ihrer Registratur, die übrigens genau in dieser Zeit im Kontext der Ausgleichsverhandlungen, das heißt der veränderten Vorstellungen von Staatlichkeit, an Bedeutung gewann und neu zu ordnen war. Die Konflikte der Herrschaftsvertreter wurden über Veränderungen des Raums ausgetragen, indem beispielsweise ‘fremdes، Gebiet mit einem Anbau, der über die eigenen Grenzen hinausreichte, besetzt wurde. Das Argument einer unbefugten Neuerung war nur glaubhaft, wenn man auf Verträge und bisherige Gewohnheiten verweisen konnte. 
Daher führte einer der ersten Wege, um sich für den Protest und Schlagabtausch zu rüsten, stets ins eigene Archiv.

Als sich die Raumvorstellungen des Regierungszentrums in der zweiten Hälfte des 18. Jahrhunderts zu ändern begannen, zogen die obersten Behörden und die lokalen Herrschaftsvertreter sozusagen an einem Strang. Die Raumvorstellungen der Beamten im Tal blieben also offensichtlich gleich. In ihrem Hüten und Wahren der Grenzen und der damit verbundenen Hoheitsrechte sahen sie sich nun durch das Agieren der Regierung - durch das Auflösen der Enklaven und die eindeutigere Bestimmung der Zuständigkeiten - wahrscheinlich gestärkt.

Die Raumkonzepte der Talbevölkerung, soweit sie in den Quellen greifbar werden, waren, wie zu erwarten, eng mit dem alltäglichen Raumhandeln verwoben. Ihr Raumbild von den Herrschaftsbereichen Tiroler und Salzburger Zuständigkeit scheint offen gewesen zu sein und ähnelte dabei dem anfänglichen Bild des Regierungszentrums. Die Menschen überschritten in der Erledigung ihrer Agenden selbstverständlich Territorialgrenzen, und an den Rändern gestaltete sich das Raumbild unbestimmt. Wenn es allerdings um Einschnitte in ihre Raumpraktiken ging oder sie sich Vorteile verschaffen wollten - etwa im Handel und Gewerbe -, dann wird deutlich, dass ihre Raumvorstellungen sehr wohl Grenzen kannten, die in diesem Fall wichtiger wurden und auch genutzt werden konnten. Mit den Veränderungen nach 1800 - wie am Beispiel der territorialen und pfarrlichen Umordnung der
Finsinger gezeigt - konfrontiert, waren sie gezwungen, ihre Raumkonzeption zu modifizieren. Von beiden Herrschaften zur Steuerleistung und Nutzung der kirchlichen Einrichtungen aufgefordert, passten sie - in einem Übergangszeitraum - ihr Raumbild und Raumhandeln an und erweiterten es.

Da jedoch für eine Bevölkerung - zudem in Zeiten der zunehmenden Abschließsung von Herrschaftsterritorien auf Dauer keine Zuordnung zu zwei Herrschaftsbereichen möglich sein konnte, war es nur eine Frage der Zeit, bis diese Praxis offenkundig und zum Problem wurde. Dieses Beispiel führte zugleich den Herrschaftsbeamten und dem Regierungszentrum vor Augen, dass die Umsetzung von idealen, abgeschlossenen Raumvorstellungen in die Praxis dann an ihre `Grenzen`stieß, wenn Räume wirtschaftlich so verflochten waren, dass eine völlige Abtrennung nur zu Lasten der Menschen und ihrer Existenz und damit in weiterer Folge auch der wirtschaftlichen Prosperität des Landes ging. Zugleich erbrachte der zunehmend präzisere Blick auf die Grenzen nicht das erhoffte Ergebnis einer klaren Scheidung von diesseitigem und jenseitigem Gebiet. Vermeintlich klar definierte Grenzlinien erschienen bei genauem Hinschauen diffus, statt einer deutlichen Zuordnung von Bevölkerung, Raum und Herrschaft machten sie neuerliche Aushandlungen und Kommunikation nötig. Damit verbanden sie, wie eingangs mit Vilém Flusser ausgeführt, die Territorien viel mehr miteinander, als dass sie sie voneinander trennten. 
A B E N DSTEIN, David: Der Streit um die Bergwerke im Zillertal zwischen Salzburg und Tirol bis 1535. Analyse, Edition und Behandlung der Thematik im Unterricht, unpublizierte Diplomarbeit, Universität Innsbruck 2014. B E H R I S C H, Lars: „Vermessen, Zählen, Berechnen des Raums im 18. Jahrhundert«, in: Lars Behrisch (Hg.): Vermessen, Zählen, Berechnen. Die politische Ordnung des Raums im 18. Jahrhundert, Frankfurt am Main 2006, S. 7-25. B I C K N E L L, Lisa / C O N R D , Benjamin / P E T E R S E N, Hans-Christian (Hg.): Kommunikation über Grenzen. Polen als Schauplatz transnationaler Akteure von den Teilungen bis heute, Berlin 2013. C н R I S T M A N N , Gabriela B.: »Das theoretische Konzept der kommunikativen Raum(re)konstruktion«, in: Gabriela B. Christmann (Hg.): Zur kommunikativen Konstruktion von Räumen. Theoretische Konzepte und empirische Analysen, Wiesbaden 2016, S. 89-117. D u H A M E L LE, Christophe / K O S SERT, Andreas / S T R U C K, Bernhard (Hg.): Grenzregionen. Ein europäischer Vergleich vom 18. bis zum 20. Jahrhundert, Frankfurt am Main 2007. D ü N N E , Jörg: „Die Karte als Operations- und Imaginationsmatrix. Zur Geschichte eines Raummediums«, in: Jörg Döring / Tristan Thielmann (Hg.): Spatial Turn. Das Raumparadigma in den Kultur- und Sozialwissenschaften, Bielefeld 2008, S. 49-69. F I S C H E R, Vladimir et al. (Hg.): Räume und Grenzen in Österreich-Ungarn 18671918. Kulturwissenschaftliche Annäherungen, Tübingen 2010. F L U S S E R, Vilém: Edition Flusser, Bd. 9: Zwiegespräche. Interviews 1967-1991, hg. von Klaus Sander, Göttingen 1996. • Kommunikologie weiter denken. Die `Bochumer Vorlesungen<, hg. von Silvia Wagnermaier / Siegfried Zielinski, Frankfurt am Main 2009. F o R S T E R, Ellinor: »'[...] daß bey weitem nicht alles salzburgischer Boden ist, was [...] gelb gemahlt ist. Aneignung von Land und Rechten durch Visualisierung auf geografischen Karten von Salzburg und Tirol im ausgehenden 18. und beginnenden 19. Jahrhundert«, in: Niels Grüne / Claus Oberhauser ( $\mathrm{Hg}$.): Jenseits des Illustrativen. Visuelle Medien und Strategien politischer Kommunikation, Göttingen 2015, S. 227-247. • / M E R T E L SEDER, Bernhard: Das Zillertal sso sehr durchkreuzet und vermischt،. Geschichten von der Grenze 1750-1816, Innsbruck 2016. F R A N ç o I S, Étienne / S E I FARTH, Jörg / S TRUCK, Bernhard (Hg.): Die Grenze als Raum, Erfahrung und Konstruktion. Deutschland, Frankreich und Polen vom 17. bis zum 20. Jahrhundert, Frankfurt am Main 2007. F R E I S T , Dagmar: »Einleitung: Staatsbildung, lokale Herrschaftsprozesse und kultureller Wandel in der Frühen Neuzeit«, in: Ronald G. Asch / Dagmar Freist (Hg.): Staatsbildung als kultureller Prozess. Strukturwandel und Legitimation von Herrschaft in der Frühen Neuzeit, Köln 2005, S. 1-47. F R I E D R I C H , Margret: "Herrschaftsverdichtung fernab vom Zentrum: Probleme der Implementierung neuer Vorstellungen und Machtrelationen am Beispiel Tirols«, in: Transylvanian Review 23/Supplement 2 (2014), S. 105-123. G A N Z E N M Ü L L E R, Jörg / Tö N S M EYER, Tatjana: „Einleitung: Vom Vorrücken des Staates in die Fläche. Ein europäisches Phänomen des langen 19. Jahrhunderts«, in: Jörg Ganzenmüller / Tatjana Tönsmeyer (Hg.): Vom Vorrücken des Staates in die Fläche. Ein europäisches Phänomen des langen 19. Jahrhunderts, Köln 2016, S. 7-31. G ePPERT, Alexander C. T. / JenSEN, Uffa / Weinhold, Jörn (Hg.): Ortsgespräche. Raum und Kommunikation im 19. und 20. Jahrhundert, Bielefeld 2005. G U L D I N , Rainer: »Ineinandergreifende graue Zonen. Vilém Flussers Bestimmung der Grenze als Ort der Begegnung«, in: Christoph Kleinschmidt / Christine Hewel (Hg.): Topographien der Grenze. Verortungen einer kulturellen, politischen und ästhetischen Kategorie, Würzburg 2011, S. 39-48. H A S L I N G E R, Peter (Hg.): Grenze im Kopf. Beiträge zur Geschichte der Grenze in Ostmitteleuropa, Frankfurt am Main 1999. H E I N D L, Waltraud et al. (Hg.): Grenze und Staat. Paßwesen, Staatsbürgerschaft, Heimatrecht und Fremdengesetzgebung in der österreichischen Monarchie 1750-1867, Wien 2000. K L E T K E, G. M.: Die Staats-Verträge des Königreichs Bayern in Bezug auf Justiz-, Polizei-, Administrations-, Landeshoheits-, Territorial- und Grenz-; Bundes-, Kirchen-, Militair-, Preß- und Nachdrucks-; Flußschifffahrts-, Post-, Eisenbahn-,
Telegraphen- und Münz-Angelegenheiten. Von 1806 bis einschließlich 1858 systematisch und chronologisch zusammengestellt, Regensburg 1860. Krol L, Frank-Lothar / Ř E Z Ík, Miloš / M u N Ke, Martin (Hg.): Sachsen und Böhmen. Perspektiven ihrer historischen Verflechtung, Berlin 2014. Lö w, Martina: Raumsoziologie, Frankfurt am Main 2001. M E t z L E R, Gabriele / W I L D T, Michael (Hg.): Über Grenzen. 48. Deutscher Historikertag in Berlin 2010. Berichtsband, Göttingen 2012. Ř E Z N Í K, Miloš (Hg.): Grenzraum und Transfer. Perspektiven der Geschichtswissenschaft in Sachsen und Tschechien, Berlin 2007. R I E D M A N N, Josef: "Zillertal«, in: Fritz Koller / Erich Marx (Hg.): Das größere Salzburg, Salzburg 2018 [im Druck]. R o L L, Christine / P OHLE, Frank / M y R Z E K, Matthias (Hg.): Grenzen und Grenzüberschreitungen. Bilanz und Perspektiven der Frühneuzeitforschung, Köln 2010. S с н E N K, Frithjof Benjamin: "Mental Maps. Die Konstruktion von geographischen Räumen in Europa seit der Aufklärung», in: Geschichte und Gesellschaft. Zeitschrift für Historische Sozialwissenschaft 28 (2002), S. 485-493. S I E G E L, Heinrich / T OMASCHEK, Karl: Österreichische Weistümer, Bd. 1: Die salzburgischen Taidinge, Wien 1870. S т o Lz, Otto: »Ein Salzburgischer Protest gegen Peter Anichs >Atlas Tyrolensis« «, in: Forschungen und Mitteilungen zur Geschichte Tirols und Vorarlbergs 9 (1912), S. 118-133. • Geschichtskunde des Zillertales, Innsbruck 1949. T A N T N E R, Anton: Ordnung der Häuser, Beschreibung der Seelen. Hausnummerierung und Seelenkonskription in der Habsburgermonarchie, Innsbruck 2007. TE U SCHER, Simon: Erzähltes Recht. Lokale Herrschaft, Verschriftlichung und Traditionsbildung im Spätmittelalter, Frankfurt am Main 2007. T н о s S, Hendrik (Hg.): Mitteleuropäische Grenzräume, Berlin 2006. T o D o R o v A, Maria: Imagining the Balkans, New York 1997. W E R N E R, Michael / Z I M M E R M A N N , Bénédicte: "Vergleich, Transfer, Verflechtung. Der Ansatz der ıHistoire croisée` und die Herausforderung des Transnationalen«, in: Geschichte und Gesellschaft. Zeitschrift für Historische Sozialwissenschaft 28 (2002), S. 607-636. W o L F F , Larry: Inventing Eastern Europe. The Map of Civilization on the Mind of the Enlightenment, Stanford 1994. 
1 Das reichsunmittelbare Erzstift wurde 1803 - zusammen mit der Reichspropstei Berchtesgaden, dem Hochstift Eichstätt und Teilen des Hochstifts Passau - zu einem weltlichen Kurfürstentum umgestaltet. Von 1806 bis 1809 gehörte es zur österreichischen Herrschaft. Nach der französischen Besetzung von 1809/1810 folgte bis 1816 die staatsrechtliche Zugehörigkeit zu Bayern, 1816 kam Salzburg wiederum und dieses Mal dauerhaft zu Österreich.

2 Die Grafschaft Tirol unterstand seit 1363 habsburgischer Herrschaft und wurde bis in die zweite Hälfte des 17. Jahrhunderts von eigenen Linien regiert. Ab diesem Zeitpunkt verwalteten von Wien eingesetzte Gouverneure das Land.

3 Vilém Flusser: Kommunikologie weiter denken. Die ıBochumer Vorlesungen«, hg. von Silvia Wagnermaier / Siegfried Zielinski, Frankfurt am Main 2009, S. 244; zu Flussers Grenzbegriff vgl. Rainer Guldin: "Ineinandergreifende graue Zonen. Vilém Flussers Bestimmung der Grenze als Ort der Begegnung«, in: Christoph Kleinschmidt / Christine Hewel (Hg.): Topographien der Grenze. Verortungen einer kulturellen, politischen und ästhetischen Kategorie, Würzburg 2011, S. 39-48.

4 Vilém Flusser: Edition Flusser, Bd. 9: Zwiegespräche. Interviews 1967-1991, hg. von Klaus Sander, Göttingen 1996, S. 62.

5 Ellinor Forster / Bernhard Mertelseder: Das Zillertal sso sehr durchkreuzet und vermischt‘. Geschichten von der Grenze 1750-1816, Innsbruck 2016, S. 11-27; Josef Riedmann: »Zillertal«, in: Fritz Koller / Erich Marx (Hg.): Das größere Salzburg, Salzburg 2018 [im Druck].

6 Flusser: Kommunikologie, S. 244.

7 Flusser: Zwiegespräche, S. 96.

8 Zu den Verträgen von 1533 und 1690 bzw. 1699 vgl. Forster / Mertelseder: Zillertal, S. 16f.; zur gemeinsamen Verwaltung der Bergwerke vgl. David Abendstein: Der Streit um die Bergwerke im Zillertal zwischen Salzburg und Tirol bis 1535. Analyse, Edition und Behandlung der Thematik im Unterricht, unpublizierte Diplomarbeit, Universität Innsbruck 2014.

9 Forster / Mertelseder: Zillertal, S. 60-67; Riedmann: „Zillertal«.

10 Forster / Mertelseder: Zillertal, S. 53-59.

11 Flusser: Zwiegespräche, S. 95.

12 Tiroler Landesarchiv (Innsbruck) [TLA], Ältere Grenzakten, Fasz. 25/7, "Oesterreichisch Salzburgische Verhandlungen zur Arrondierung der Territorialverhältnisse im Zillertal 1781-1793«, Gottfried von Moll: »Hochfürstlich Salzburgische Gegenerklärung auf die von Kaiserlich Königlichen Hof-Kanzlei anher erlassenen Vergleichs-Punkten wegen Zillerthal«, 6. 10. 1780.

13 Lars Behrisch: "Vermessen, Zählen, Berechnen des Raums im 18. Jahrhundert«, in: Lars Behrisch (Hg.): Vermessen, Zählen, Berechnen. Die politische Ordnung des Raums im 18. Jahrhundert, Frankfurt am Main 2006, S. 7-25, hier S. 16; vgl. auch Jörg Ganzenmüller / Tatjana Tönsmeyer: „Einleitung: Vom Vorrücken des Staates in die Fläche. Ein europäisches Phänomen des langen 19. Jahrhunderts«, in: Jörg Ganzenmüller / Tatjana Tönsmeyer (Hg.): Vom Vorrücken des Staates in die Fläche. Ein europäisches Phänomen des langen 19. Jahrhunderts, Köln 2016, S. 7-31.

14 Anton Tantner: Ordnung der Häuser, Beschreibung der Seelen. Hausnummerierung und Seelenkonskription in der Habsburgermonarchie, Innsbruck 2007, S. 59.

15 Ellinor Forster: »'[...] daß bey weitem nicht alles salzburgischer Boden ist, was [...] gelb gemahlt ist. Aneignung von Land und Rechten durch Visualisierung auf geografischen Karten von Salzburg und Tirol im ausgehenden 18. und beginnenden 19. Jahrhundert«, in: Niels Grüne / Claus Oberhauser (Hg.): Jenseits des Illustrativen. Visuelle Medien und Strategien politischer Kommunikation, Göttingen 2015, S. 227-247.
16 Siehe dazu die Einleitung zu diesem Band; sowie Dagmar Freist: »Einleitung: Staatsbildung, lokale Herrschaftsprozesse und kultureller Wandel in der Frühen Neuzeit«, in: Ronald G. Asch / Dagmar Freist (Hg.): Staatsbildung als kultureller Prozess. Strukturwandel und Legitimation von Herrschaft in der Frühen Neuzeit, Köln 2005, S. 1-47.

17 Martina Löw: Raumsoziologie, Frankfurt am Main 2001, S. 67, 131, 153-166.

18 Gabriela B. Christmann: „Das theoretische Konzept der kommunikativen Raum(re)konstruktion«, in: Gabriela B. Christmann (Hg.): Zur kommunikativen Konstruktion von Räumen. Theoretische Konzepte und empirische Analysen, Wiesbaden 2016, S. 89-117.

19 Die Bedeutung des Handelns für die Raumkonstruktion betont auch Löw: Raumsoziologie, S. 67.

201754 waren die Kreisämter als Zwischeninstanz eingeführt worden, allerdings lief die Kommunikation in den ersten Jahrzehnten noch an ihnen vorbei; Margret Friedrich: »Herrschaftsverdichtung fernab vom Zentrum: Probleme der Implementierung neuer Vorstellungen und Machtrelationen am Beispiel Tirols«, in: Transylvanian Review 23/Supplement 2 (2014), S. 105-123.

21 Über die Regierungswechsel hinweg veränderten sich auch die Bezeichnungen der zentralen Behörden: Unter österreichischer Herrschaft wurde in Salzburg eine Landesregierung installiert, im Kontext des Königreichs Bayern gab es sowohl in Innsbruck als auch in Salzburg zunächst ein Hofkommissariat, das nach einigen Monaten durch ein Generallandeskommissariat ersetzt wurde.

22 Zur Einordnung der Forschung siehe die Einleitung zu diesem Band.

23 Waltraud Heindl et al. (Hg.): Grenze und Staat. Paßwesen, Staatsbürgerschaft, Heimatrecht und Fremdengesetzgebung in der österreichischen Monarchie 1750-1867, Wien 2000.

24 Peter Haslinger (Hg.): Grenze im Kopf. Beiträge zur Geschichte der Grenze in Ostmitteleuropa, Frankfurt am Main 1999; Vladimir Fischer et al. (Hg.): Räume und Grenzen in Österreich-Ungarn 1867-1918. Kulturwissenschaftliche Annäherungen, Tübingen 2010. Einen speziellen Fokus auf die gemeinsame Geschichte der Lausitzen legen etwa Miloš Řezník (Hg.): Grenzraum und Transfer. Perspektiven der Geschichtswissenschaft in Sachsen und Tschechien, Berlin 2007; Frank-Lothar Kroll / Miloš Řezník / Martin Munke (Hg.): Sachsen und Böhmen. Perspektiven ihrer historischen Verflechtung, Berlin 2014.

25 Étienne François / Jörg Seifarth / Bernhard Struck (Hg.): Die Grenze als Raum, Erfahrung und Konstruktion. Deutschland, Frankreich und Polen vom 17. bis zum 20. Jahrhundert, Frankfurt am Main 2007; Christophe Duhamelle / Andreas Kossert / Bernhard Struck (Hg.): Grenzregionen. Ein europäischer Vergleich vom 18. bis zum 20. Jahrhundert, Frankfurt am Main 2007.

26 Hendrik Thoß (Hg.): Mitteleuropäische Grenzräume, Berlin 2006; Lisa Bicknell / Benjamin Conrad / Hans-Christian Petersen (Hg.): Kommunikation über Grenzen. Polen als Schauplatz transnationaler Akteure von den Teilungen bis heute, Berlin 2013.

27 Gabriele Metzler / Michael Wildt (Hg.): Über Grenzen. 48. Deutscher Historikertag in Berlin 2010. Berichtsband, Göttingen 2012; Christine Roll / Frank Pohle / Matthias Myrczek (Hg.): Grenzen und Grenzüberschreitungen. Bilanz und Perspektiven der Frühneuzeitforschung, Köln 2010.

28 Michael Werner / Bénédicte Zimmermann: „Vergleich, Transfer, Verflechtung. Der Ansatz der >Histoire croisée derung des Transnationalen«, in: Geschichte und Gesellschaft. Zeitschrift für Historische Sozialwissenschaft 28 (2002), S. 607-636.

29 Als Überblick über die Forschung zu ımental maps` vgl. Frithjof Benjamin Schenk: »Mental Maps. Die Konstruktion von geographischen Räumen in Europa seit der Aufklärung", in: Geschichte und Gesellschaft. Zeitschrift für Historische Sozialwissenschaft 28 (2002), 
S. 485-493. Grundlegende Untersuchungen sind etwa Maria Todorova: Imagining the Balkans, New York 1997; Larry Wolff: Inventing Eastern Europe. The Map of Civilization on the Mind of the Enlightenment, Stanford 1994.

30 Allgemein (nicht speziell zu Grenzen) vgl. Alexander C. T. Geppert / Uffa Jensen / Jörn Weinhold (Hg.): Ortsgespräche. Raum und Kommunikation im 19. und 20. Jahrhundert, Bielefeld 2005.

31 Das Landrecht wurde hier sowohl als eine Sammlung der Statuten als auch als "Landtaiding" verstanden, als die jährliche Versammlung zur Rechtsprechung; Heinrich Siegel / Karl Tomaschek: Österreichische Weistümer, Bd. 1: Die salzburgischen Taidinge, Wien 1870, S. 317-325. Dazu vgl. Simon Teuscher: Erzähltes Recht. Lokale Herrschaft, Verschriftlichung und Traditionsbildung im Spätmittelalter, Frankfurt am Main 2007.

32 Siegel / Tomaschek: Taidinge, S. $317 \mathrm{f}$.

33 TLA, Handschriften, Nr. 3845, Verträge vom 1. 12. 1533, 9. 12. 1690, 29. 4. 1699; Salzburger Landesarchiv (Salzburg) [SLA], Hofrat [HR], Kropfsberg, Nr. 94, "Irrung mit Tirol die Setzung der Marchsteine betr.«, 1690/1715.

34 TLA, Karten und Pläne, Nr. 720, Karte von Wolfgang Hagenauer, Salzburg 1766; Nr. 2867, Karte von Johann Anton von Erlach, Schwaz 1766. Gegenüberstellung der Kartenlegenden sowie weitere Abbildungen und Ausschnitte der Karten bei Forster / Mertelseder: Zillertal, Einband vorne, S. 28-48, 80.

35 Forster: »Aneignung von Land und Rechten«, S. 227-229; Otto Stolz: "Ein Salzburgischer Protest gegen Peter Anichs `Atlas Tyrolensis«", in: Forschungen und Mitteilungen zur Geschichte Tirols und Vorarlbergs 9 (1912), S. 118-133; zur Interpretation von Landkarten im 18. Jahrhundert vgl. Jörg Dünne: „Die Karte als Operations- und Imaginationsmatrix. Zur Geschichte eines Raummediums«, in: Jörg Döring / Tristan Thielmann (Hg.): Spatial Turn. Das Raumparadigma in den Kultur- und Sozialwissenschaften, Bielefeld 2008, S. 49-69.

36 TLA, Handschriften, Nr. 3845, Vertrag von 1690 in der ratifizierten Form von 1699, Punkt 3, fol. 12v.

37 TLA, Ältere Grenzakten, Fasz. 25/7, Johann Franz Strobl: „Gutachten über die zwischen Tyrol und Salzburg bestehenden vorgehenden Differenzen und derselben Beylegung«, 11. 7. 1793, § IX.

38 TLA, Ältere Grenzakten, Fasz. 25/7, Schreiben des Registraturdirektors Gassler an das Gubernium in Innsbruck, 21. 2. 1791.

39 SLA, HR, Kropfsberg, Nr. 143, Schreiben des Pflegers von Zell und Fügen Gottfried von Moll an den Hofrat in Salzburg, 8. 10. 1793.

40 SLA, HR, Kropfsberg, Nr. 143, Grenzbesichtigungsprotokoll 1798

41 SLA, HR, Kropfsberg, Nr. 143, Grenzbesichtigungsprotokoll 1793, Markierungspunkt LXXXVIII.

42 SLA, HR, Kropfsberg, Nr. 143, Grenzbesichtigungsprotokoll 1793, Markierungspunkt VI.

43 SLA, HR, Kropfsberg, Nr. 143, Grenzbesichtigungsprotokoll 1793, Markierungspunkte IX »Furthbrunn«, XXVI »Eyrengelenk, Faltenstain, Leitenjoch«.

44 SLA, HR, Kropfsberg, Nr. 143, Grenzbesichtigungsprotokoll 1798, Markierungspunkt XLI. Die strittige Bezeichnung war der "Schwarzbrunn«.

45 SLA, HR, Kropfsberg, Nr. 143, Grenzbesichtigungsprotokoll 1798, Markierungspunkt VI.

46 SLA, HR, Kropfsberg, Nr. 143, Grenzbesichtigungsprotokoll 1798, Markierungspunkt IX

47 SLA, HR, Kropfsberg, Nr. 143, Grenzbesichtigungsprotokoll 1798, beim Markierungspunkt LXVIII.

48 SLA, HR, Kropfsberg, Nr. 143, Grenzbesichtigungsprotokoll 1798, beim Markierungspunkt CIV.

49 SLA, HR, Fügen, Nr. 2, "Die Grenze, der Sommerlange Zaun im Zillertal, Irrung mit Tirol«, 1679.
50 SLA, HR, Fügen, Nr. 2, Befragung von Martin Hochmuet, Fügen, 4. 10. 1679.

51 SLA, HR, Fügen, Nr. 15, »Irrung mit Tirol wegen des Wirtshauses in Rottenburg und des Brotverkaufes der Rottenburger Bäcker«, 1757; Kropfsberg, Nr. 102, »Beschwerde der Innsbrucker Repräsentation gegen die recesswidrigen Jurisdiktionseingriffe der Pfleggerichte Fügen und Kropfsberg", 1756; vgl. Otto Stolz: Geschichtskunde des Zillertales, Innsbruck 1949, S. 202.

52 Das Wissen um die weiteren Zusammenhänge hing von der jeweiligen Person des Pflegers ab. Gottfried von Moll, der von 1764 bis 1795 beide Salzburger Pflegen im Zillertal verwaltete, war etwa zugleich der Beauftragte für die Ausgleichsverhandlungen. Daher kumulierte sich bei ihm das Wissen um die lokalen Verhältnisse sowie den jeweiligen Verhandlungsstand und die gegnerischen Argumentationsstrategien.

53 SLA, HR, Fügen, Nr. 30, Schreiben des Pflegers von Zell und Fügen Gottfried von Moll an den Hofrat in Salzburg, 24. 1. 1785; Österreichisches Staatsarchiv (Wien) [ÖStA], Haus-, Hof- und Staatsarchiv [HHStA], Handschriften, W 199, Bd. 2, Heinrich Freiherr von Reigersberg: »Beschreibung des Zillertals«, 1789, fol. 245r-v.

54 SLA, HR, Kropfsberg, Nr. 109, Schreiben des Pflegers von Rottenburg Josef Strolz an den Pfleger von Zell und Fügen Gottfried von Moll, 31. 8. 1769.

55 SLA, HR, Fügen, Nr. 15, Beeidigte Aussage von Barthlme Gwerchner, Fügen, 7. 1. 1757.

56 SLA, HR, Fügen, Nr. 15, Schreiben des Pflegers von Fügen Carl Leopold Lürzer an den Erzbischof in Salzburg, 18. 4. 1757.

57 SLA, HR, Fügen, Nr. 15, Schreiben der Wirte und Bierzapfler von Fügen an den Erzbischof in Salzburg, O. D. [nach November 1756].

58 SLA, HR Fügen, Nr. 15, Abschrift des Attests des Dechants von Fügen Franz Wilhelm Freiherr von Enzenberg, 16. 12. 1753.

59 SLA, HR, Fügen, Nr. 15, Konzept des Hofrats in Salzburg an den Pfleger von Fügen Carl Leopold Lürzer, 6. 11. 1756.

60 SLA, HR, Fügen, Nr. 15, Schreiben des Pflegers von Rottenburg Michael Ignaz von Löffler an den Pfleger von Fügen Carl Leopold Lürzer, 20. 12. 1756.

61 SLA, HR, Fügen, Nr. 15, Schreiben des Pflegers von Fügen Carl Leopold Lürzer an den Pfleger von Rottenburg Michael Ignaz von Löffler, 4. 1. 1757.

62 SLA, HR, Fügen, Nr. 15, Schreiben des Pflegers von Fügen Carl Leopold Lürzer an den Erzbischof in Salzburg, 18. 4. 1757.

63 SLA, HR, Fügen, Nr. 15, Schreiben des Pflegers von Fügen Carl Leopold Lürzer an den Erzbischof in Salzburg, 18. 4. 1757.

64 SLA, HR, Fügen, Nr. 15, Schreiben des Pflegers von Fügen Carl Leopold Lürzer an den Pfleger von Rottenburg Michael Ignaz von Löffler, 4. 1. 1757

65 SLA, HR, Fügen, Nr. 15, Schreiben der Repräsentation und Kammer in Innsbruck an den Erzbischof von Salzburg, 14. 3. 1757.

66 SLA, HR, Fügen, Nr. 15, Schreiben des Pflegers von Rottenburg Michael Ignaz von Löffler an den Pfleger von Fügen Carl Leopold Lürzer, 20. 12. 1756.

67 SLA, HR, Fügen, Nr. 15, Schreiben des Pflegers von Fügen Carl Leopold Lürzer an den Pfleger von Rottenburg Michael Ignaz von Löffler, 4. 1. 1757.

68 SLA, HR, Fügen, Nr. 15, Schreiben des Pflegers von Zell Johann Anton Pircker an den Erzbischof in Salzburg, 23. 11. 1761

69 SLA, HR, Fügen, Nr. 15, Schreiben des Pflegers von Zell Johann Anton Pircker an den Erzbischof in Salzburg, 26. 11. 1761; Schreiben des Amtsschreibers von Fügen Rochus Braun an den Pfleger von Zell Johann Anton Pirker, 24. 11. 1761.

70 SLA, HR, Fügen, Nr. 15, Konzept des Hofrats in Salzburg an den Pfleger von Fügen Carl Leopold Lürzer, 21. 8. 1761. 
71 SLA, HR, Fügen, Nr. 15, Schreiben der Repräsentation und Kammer in Innsbruck an den Erzbischof von Salzburg, 14. 3. 1757.

72 SLA, HR, Fügen, Nr. 15, Schreiben der Repräsentation und Kammer in Innsbruck an den Erzbischof von Salzburg, 14. 3. 1757.

73 SLA, HR, Fügen, Nr. 15, Konzept des Hofrats in Salzburg an den Pfleger in Fügen, 22. 10. 1742.

74 SLA, HR, Fügen, Nr. 15, Schreiben des Pflegers von Fügen Carl Leopold Lürzer an den Erzbischof in Salzburg, 18. 4. 1757.

75 SLA, HR, Fügen, Nr. 15, Konzept des Hofrats in Salzburg an den Pfleger in Fügen, 15. 1. 1748.

76 SLA, HR, Fügen, Nr. 15, Schreiben des Pflegers von Fügen Carl Leopold Lürzer an den Erzbischof in Salzburg, 18. 4. 1757.

77 Nachdem nach 1764 die lokalen Beamten gewechselt hatten, kam es nach mehrmaligen Ausgleichsversuchen 1769 zu einem vermittelnden Treffen beim Dechant im Pfarrhof von Fügen, das jedoch auch keine dauerhafte Lösung herbeiführte. Neuerlich wurde über die Aufhebung der Ausschankberechtigungen, der Verbote des Besuchs sowie der Biereinfuhr gestritten. Der Hofrat in Salzburg blieb auch in diesem Fall bei der Mahnung zur nachbarlichen Eintracht; SLA, HR, Kropfsberg, Nr. 109, Schreiben des Pflegers von Rottenburg Josef Strolz an den Pfleger von Zell und Fügen Gottfried von Moll, 31. 8. 1769; Schreiben des Pflegers von Zell und Fügen Gottfried von Moll an den Erzbischof in Salzburg, 4. 9. 1769.

78 SLA, HR, Kropfsberg, Nr. 113, Konzept des Hofrats in Salzburg an den Pfleger von Zell und Fügen Gottfried von Moll, 9. 10. 1773.

79 SLA, HR, Fügen, Nr. 15, Schreiben der Repräsentation und Kammer in Innsbruck an den Erzbischof von Salzburg, 14. 3. 1757.

80 SLA, HR, Fügen, Nr. 15, Schreiben des Pflegers von Fügen Carl Leopold Lürzer an den Erzbischof in Salzburg, 18. 4. 1757.

81 SLA, HR, Kropfsberg, Nr. 128, "Die vom Gubernium in Innsbruck angebotene Aufhebung der das Ziller- und Brixental umgebenden k. k. Zollstätten«, 1783

82 Art. 15 des Friedens von Pressburg, 26. 12. 1805, abgedruckt in: G. M. Kletke: Die Staats-Verträge des Königreichs Bayern in Bezug auf Justiz-, Polizei-, Administrations-, Landeshoheits-, Territorialund Grenz-; Bundes-, Kirchen-, Militair-, Preß- und Nachdrucks-; Flußschifffahrts, Post-, Eisenbahn-, Telegraphen- und Münz-Angelegenheiten. Von 1806 bis einschließlich 1858 systematisch und chronologisch zusammengestellt, Regensburg 1860, S. 161-168, hier S. $165 f$.

83 TLA, Cattanea, Nr. 768, z. B. „Vortrag über die differenten Landesund Forstgrenzen«, Salzburg, 19. 7. 1806.

84 ÖStA, HHStA, Österreichische Akten [ÖA], Salzburg [Sbg], Karton 15, Fasz. 22, Nr. II, „Gutachten über die Modalitäten zur Vollziehung der vom Kaiserlich-Königlichen Hof beschlossenen Regulirung des Salzburgischen Metropolitan-Kirchen-Sprengels«, Salzburg 2. 6. 1807.

85 ÖStA, HHStA, ÖA, Sbg, Karton 15, Fasz. 22, Nr. VII, Verhör mit Maria Pfisterin, 30. 6. 1808; weiters vgl. Verhör mit Peter Pircher, 27. 6. 1808.

86 ÖStA, HHStA, ÖA, Sbg, Karton 15, Fasz. 22, Nr. VII, Verhör mit Peter Pircher, 27. 6. 1808.

87 ÖStA, HHStA, ÖA, Sbg, Karton 15, Fasz. 22, Nr. VII, Verhör mit Ursula Höllwarthin, 30. 6. 1808

88 ÖStA, HHStA, ÖA, Sbg, Karton 15, Fasz. 22, Nr. VII, Verhör mit Peter Pircher, 27. 6. 1808.

89 ÖStA, HHStA, ÖA, Sbg, Karton 15, Fasz. 22, Nr. VII, Verhör mit Josef Widgschwentner, 30. 6. 1808.

90 ÖStA, HHStA, ÖA, Sbg, Karton 15, Fasz. 22, Nr. VII, Verhör mit Dorothea Mayrin, 30. 6. 1808.

91 ÖStA, HHStA, ÖA, Sbg, Karton 15, Fasz. 22, Nr. VII, Verhör mit Franz Rainer, 30. 6. 1808.
92 ÖStA, HHStA, ÖA, Sbg, Karton 15, Fasz. 22, Nr. VII, Verhör mit Paul Reindl, 30. 6. 1808.

93 ÖStA, HHStA, ÖA, Sbg, Karton 15, Fasz. 22, Nr. VII, Verhör mit Joseph Sandbichler, 30. 6. 1808.

94 ÖStA, HHStA, ÖA, Sbg, Karton 15, Fasz. 22, Nr. VII, Verhör mit Ursula Höllwarthin, 30. 6. 1808.

95 ÖStA, HHStA, ÖA, Sbg, Karton 15, Fasz. 22, Nr. VII, Verhör mit Joseph Sandbichler, 30. 6. 1808.

96 ÖStA, HHStA, ÖA, Sbg, Karton 15, Fasz. 22, Nr. VII, Schreiben des abgeordneten Kommissärs Trauner in Zell an die Landesregierung in Salzburg, 1. 7. 1808.

97 Siehe die Einleitung zu diesem Band. 


\section{Abstract}

In the light of the relevant terms of this issue, "state«, "space" and "administration«, this contribution considers the intertwining between representations of order, their administration, and the governance of the subjects in the sovereignty areas of Salzburg and Tyrol in the Zillertal. The different ideas of space - investigated from the perspectives of various groups of the population, of local officials, and of the government centers - changed throughout the examined period. At its beginning, the authorities in the government centers endeavored to keep the borders open for mutual exchange, whereas the local officials used their administrative tools to stage a competition with the officials of the neighboring district, e.g. by blocking and redirecting the subjects' pathways of movement. In this situation, a more open construction of space confronted a more mistrustful one aimed at enclosure and the guarding of borders. In the context of negotiations for a general border settlement between Tyrol and Salzburg, and on the basis of newly developed conceptions of a state as having a clearly defined sovereignty area, capable of being governed without any foreign influence up to its borders, the interventions of the government centers started to change. Borders were adjusted and a clear assignment of the subjects was demanded. However, the more an exact correspondence between space and sovereignty was pursued, the more obvious the impossibility of this undertaking became. Seemingly well-demarcated border lines appeared vague when regarded closely. Their official description was at odds with the subjects' construction and usage of spaces. While their spatial behaviors were determined by the norms of the sovereign centers and controlled by the administrative work of the officials, the subjects developed their own strategies for dealing with these interventions in their constructions of space and adjusting to them.

\section{About the Author}

Ellinor Forster, PhD, Senior Lecturer at the Institute of Modern and Comtemporary History, University of Linz. 20112016 holder of a position in the Elise Richter Programme (Austrian Science Fund - FWF) for the project »Changing Society through Rule and Government. Political Communication in the Territories of Ferdinand III - Tuscany, Salzburg and Wurzburg 1790-1824«, 2015/2016 Fellowship resp. Scholarship in Opava (Czech Republic) and Rome, 2008-2013 Assistant Professor at the Institute of History and European Ethnology, University of Innsbruck. 